\title{
3,5-Bis-(trifluoromethyl)phenyl sulfones in the synthesis of 3,5-disubstituted cyclopent-2-enones
}

\author{
Diego A. Alonso, Armin Babrowski, ${ }^{\dagger}$ Mónica Fuensanta, Carmen Nájera, \\ and Montserrat Varea \\ Departamento de Química Orgánica and Instituto de Síntesis Orgánica (ISO), Facultad de \\ Ciencias, Universidad de Alicante, Apartado 99, E-03080 Alicante, Spain \\ E-mail: cnajera@ua.es
}

\section{Dedicated to Professor Lutz F. Tietze}

\begin{abstract}
3,5-Bis-(trifluoromethyl)phenyl sulfones (BTFP sulfones) 1a-e, easily synthesized from 3,5-bis(trifluoromethyl)benzenethiol, react under PTC with (Z)-1,4-dichloro-2-butene to afford the cyclopentenyl sulfones 3 , which suffer further diastereoselective alkene epoxidation with MCPBA giving BTFP sulfonyl cyclopentene oxides $\mathbf{5}$ and $\mathbf{6}$ in good yields. These epoxides are convenient precursors of 3,5-disubstituted cyclopent-2-enones, which are given after epoxide ring-opening with different nucleophiles and final successive oxidation-BTFP sulfinate elimination.
\end{abstract}

Keywords: Sulfones, cyclopentenones, epoxides, dialkylation, oxidation, elimination

\section{Introduction}

The cyclopentenone structure occurs in a wide variety of biologically active compounds such as prostaglandins, pyrethroids, and steroids. Cyclopentenones are also often used as building blocks for the synthesis of other biologically active compounds owing to their suitability for further functionalization via nucleophilic 1,4-addition and reaction with electrophiles at the 2- and 5positions (Figure 1). For all these reasons, numerous synthetic methods have been developed for their preparation. Among them we can mention classical routes such as the base-catalyzed intramolecular aldol condensation of 1,4-dicarbonyl compounds, ${ }^{1}$ the intramolecular Wittig- type reactions, ${ }^{2}$ intramolecular $1,5-\mathrm{C}-\mathrm{H}$ insertions, ${ }^{3}$ and the Nazarov ${ }^{4}$ cyclization. Moreover, different transition metal-mediated approaches ${ }^{5}$ such as the Pauson-Khand reaction, ${ }^{6}$ and the Rautenstrauch rearrangement, ${ }^{7}$ have become very popular for the synthesis of this type of compounds.

\footnotetext{
${ }^{\dagger}$ Undergraduate student from the Albert-Ludwigs-Universität Freiburg.
} 


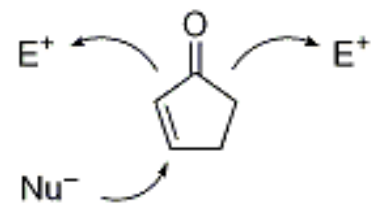

Figure 1. Cyclopentene functionalization.

We have recently shown that the 3,5-bis-(trifluoromethyl)phenyl (BTFP) group is a strong electron-withdrawing group, and that the corresponding BTFP sulfonyl group is an excellent nucleofuge in base-promoted $\beta$-elimination processes used in the stereoselective synthesis of $E$ aconitates by dialkylation under phase-transfer-catalyzed conditions followed by concomitant elimination $^{8}$ (Figure 2). 2-BTFP-sulfonyl-ethanol is an efficient protective group for carboxylic acids, which is easily removed with aqueous $\mathrm{NaHCO}_{3}$ (Figure 2). ${ }^{9}$ Also, alkyl BTFP sulfones can be used in the stereoselective synthesis of di-, tri-, and tetra-substituted olefins through the JuliaKocienski olefination ${ }^{10}$ of aliphatic and aromatic aldehydes and ketones under very simple and mild reaction conditions by using $\mathrm{KOH}$ and phosphazenes as bases ${ }^{11}$ (Figure 2).

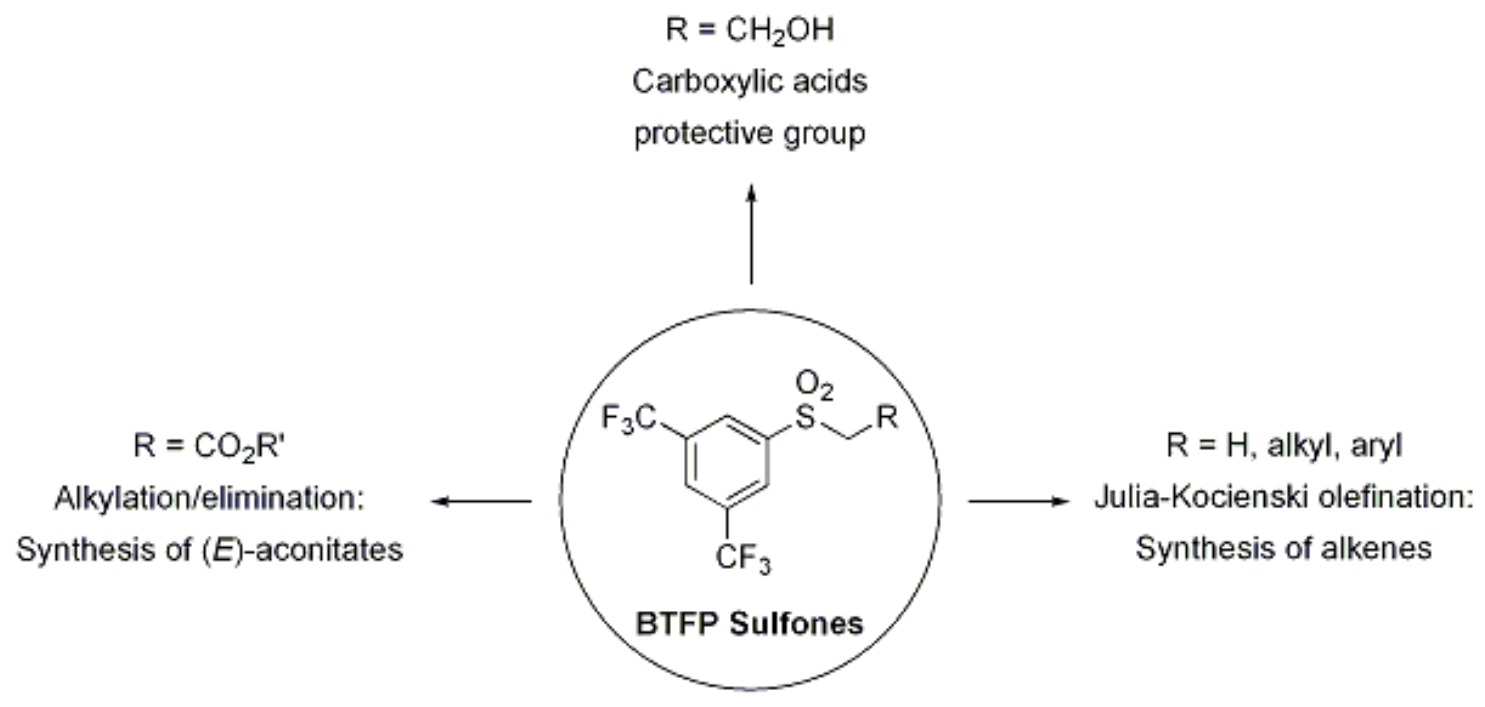

Figure 2. BTFP sulfones in organic synthesis.

Recently, functionalized 3,5-disubstituted cyclopent-2-enones have been prepared via a solidphase sulfone-linker strategy employing a phenylsulfonyl- functionalized polystyrene/ divinylbenzene resin. ${ }^{12}$ In the present study, we report on the synthesis of 3,5-disubstituted cyclopentenones, employing functionalized alkyl BTFP sulfones in a four-step synthetic strategy as depicted in the retrosynthetic Scheme 1. We assume that the dialkylation step should be easily performed under PTC $^{8}$ and the final oxidation and $\beta$-elimination of BTFP sulfinic acid should take readily place in an in situ process. 


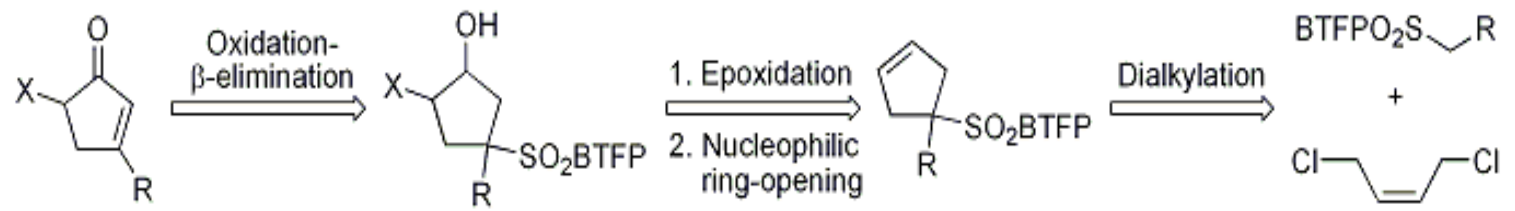

Scheme 1. Retrosynthesis of 3,5-disubstituted cyclopentenones from BTFP sulfones.

\section{Results and Discussion}

For the preparation of alkyl BTFP sulfones 1, a previously described two-step procedure was used (Scheme 2). ${ }^{8}$ The alkylation reaction of 3,5-bis-(trifluoromethyl)benzenethiol ${ }^{13}$ with alkyl bromides using $\mathrm{NaH}$ as base in $\mathrm{CH}_{3} \mathrm{CN}$ at RT afforded the corresponding sulfides 2, which were oxidized without further purification. The oxidation was performed with $30 \% \mathrm{H}_{2} \mathrm{O}_{2}$ in the presence of substoichiometric amounts of $\mathrm{MnSO}_{4} \cdot \mathrm{H}_{2} \mathrm{O}(1 \mathrm{~mol} \%)$ and a buffer solution of $\mathrm{NaHCO}_{3}{ }^{14}$ for sulfides 1a-c, or Oxone ${ }^{\circledR}$ in $\mathrm{MeOH} / \mathrm{H}_{2} \mathrm{O}$ at RT for the synthesis of sulfones $1 \mathbf{d}-\mathbf{e}$ (Scheme 2). The overall yields obtained ranged from $60 \%$ for the phosphonate derivative 1e to $93 \%$ for the benzyl sulfone 1a (Scheme 2).

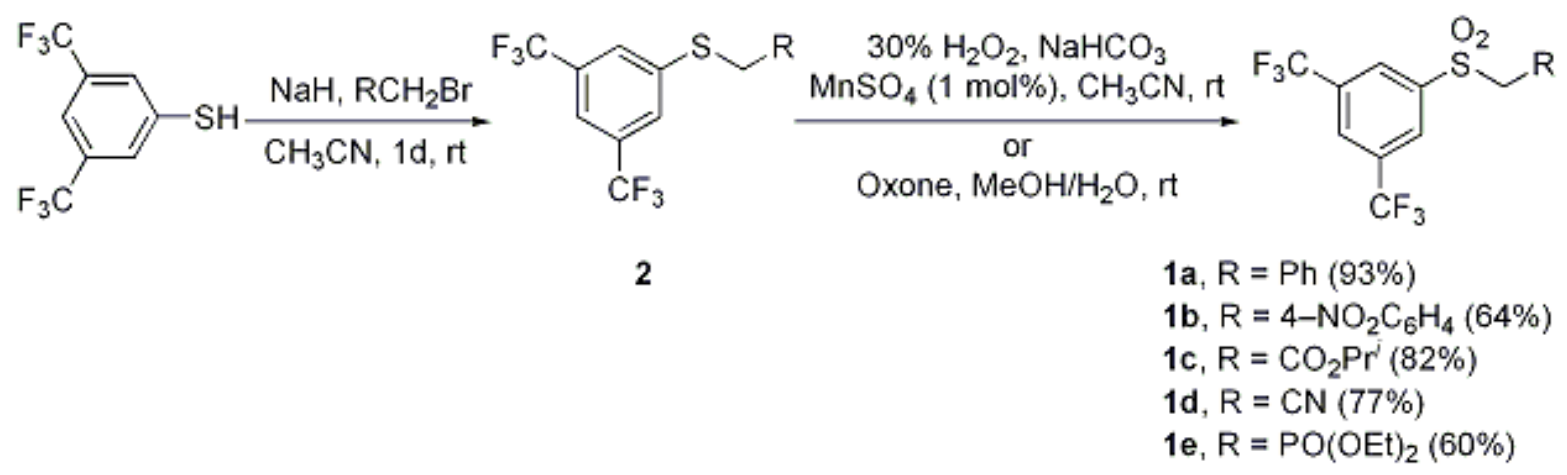

Scheme 2. Synthesis of the BTFP sulfones 1a-e.

The cyclopentenes 3a-e were prepared in good yields under very mild PTC conditions by $\alpha, \alpha-$ dialkylation reaction of the sulfone with (Z)-1,4-dichlorobut-2-ene (Scheme 3, Table 1). The benzylic sulfones 1a and $\mathbf{1 b}$ were submitted to the dialkylation process employing $\mathrm{KOH}$ as base to give the cyclopentene derivatives $\mathbf{3 a}$ and $\mathbf{3 b}$ in 76 and 58\% yield, respectively (Table 1, entries 1 and 2). In the $\alpha, \alpha$-dialkylation of BTFP sulfone $\mathbf{1 b}$ a $28 \%$ yield of the cyclopentenol $\mathbf{4}$ was also obtained as a consequence of a nucleophilic substitution of the sulfonyl group by the base. On the other hand, $\mathrm{K}_{2} \mathrm{CO}_{3}$ was the base which gave the best yields for the $\alpha, \alpha$-dialkylation reaction of the more acidic sulfones 1c-e, affording the corresponding adducts 3c-e in yields between 60 and $76 \%$ (Table 1, entries 3-5). It is worth mentioning that non-activated benzyl sulfones require much stronger bases, such as $n$-BuLi at $0^{\circ} \mathrm{C}$, to perform this $\alpha$, $\alpha$-dialkylation process with $(Z)-1,4-$ dichlorobut-2-ene. ${ }^{12}$

Epoxidation of the sulfonyl cyclopentenes 3 with MCPBA in $\mathrm{CH}_{2} \mathrm{Cl}_{2}$ at $50^{\circ} \mathrm{C}$ for $18 \mathrm{~h}$ delivered diastereoselectively the corresponding oxiranes 5-6 which were easily separated by column 
chromatography (Scheme 3, Table 1). We initially assumed that the observed major diastereomer in the epoxidation reaction corresponded to the product with the relative stereochemistry depicted in $\mathbf{6}$, since these isomers were the only products obtained in similar epoxidations performed with (phenylsulfonyl)cyclopentene derivatives. ${ }^{12,15 a}$ The formation of the major diastereomer $\mathbf{6}$ can be probably be due to steric reasons.

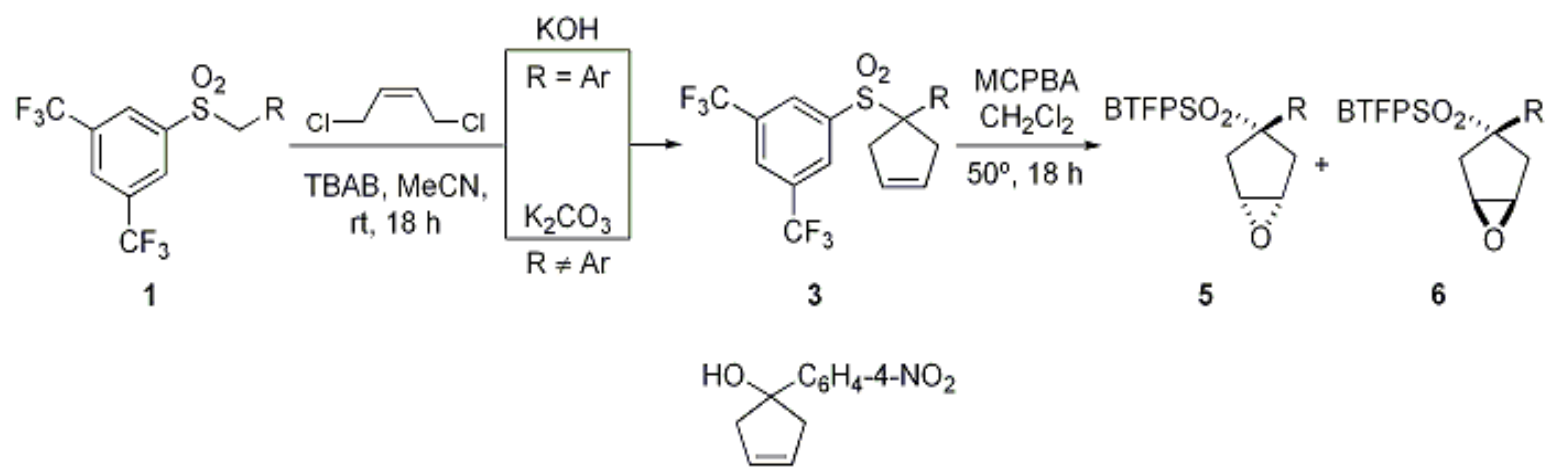

4

Scheme 3. Synthesis of BTFP sulfonyl cyclopentenes $\mathbf{3}$ and oxiranes $\mathbf{5}$ and $\mathbf{6}$.

Table 1. Synthesis of BTFP sulfonylcyclopentenes $\mathbf{3}$ and oxiranes $\mathbf{5}$ and $\mathbf{6}$

\begin{tabular}{|c|c|c|c|c|c|c|c|}
\hline \multirow[b]{2}{*}{ Entry } & \multicolumn{2}{|c|}{ BTFP sulfones } & \multirow[b]{2}{*}{ Base } & \multicolumn{2}{|c|}{ Cyclopentyl sulfones } & \multicolumn{2}{|c|}{ BTFP sulfonyl oxiranes } \\
\hline & No. & $\mathrm{R}$ & & No. & Yield (\%) ${ }^{a}$ & 5 Yield $(\%)^{b}$ & 6 Yield $(\%)^{b}$ \\
\hline 1 & 1a & $\mathrm{Ph}$ & $\mathrm{KOH}$ & 3a & 76 & $5 \mathbf{a}(9)$ & 6a (76) \\
\hline 2 & 1b & $4-\mathrm{NO}_{2} \mathrm{C}_{6} \mathrm{H}_{4}$ & $\mathrm{KOH}$ & $3 \mathbf{b}$ & $58^{c}$ & $5 \mathbf{b}(2)$ & $6 \mathbf{b}(43)$ \\
\hline 3 & 1c & $\mathrm{CO}_{2} \mathrm{Pr}^{i}$ & $\mathrm{~K}_{2} \mathrm{CO}_{3}$ & $3 c$ & 60 & $5 c(3)$ & 6c (69) \\
\hline 4 & 1d & $\mathrm{CN}$ & $\mathrm{K}_{2} \mathrm{CO}_{3}$ & 3d & 64 & $5 d(6)$ & 6d (39) \\
\hline 5 & 1e & $\mathrm{PO}(\mathrm{OEt})_{2}$ & $\mathrm{~K}_{2} \mathrm{CO}_{3}$ & $3 \mathbf{e}$ & 76 & $5 e(20)$ & 6e (47) \\
\hline
\end{tabular}

${ }^{a}$ Isolated yield after flash chromatography, based on the starting sulfones $1{ }^{b}$ Isolated yield after flash chromatography, based on the cyclopentenes $3 .{ }^{c}$ A $28 \%$ yield of 4 was also obtained.

The next step towards the synthesis of 2-cyclopentenones consisted in the nucleophilic ring opening of the sulfonyl epoxides with various nucleophiles (Scheme 4, Table 2). This study was carried out with the major epoxide diastereomers $\mathbf{6}$ in order to prepare diastereomerically pure products 7. A preliminary screening of the reaction was performed with epoxide $\mathbf{6 a}$, employing different nucleophiles. Nucleophilic ring-opening of the epoxide moiety in $\mathbf{6 a}$ with sodium azide in DMF at $100^{\circ} \mathrm{C}$ afforded the cyclopentanol 7aa in a 54\% yield (Table 2, entry 1). A higher yield (66\%) was obtained when the reaction was performed employing an excess of lithium azide (20\% aqueous solution) (Table 2, entry 2). When employing amines as nucleophiles, it was necessary to use a larger excess of the reagent, longer reaction times, and solventless conditions, as depicted for benzylamine in Table 2 entries 3 and 4 . With the purpose of reducing the reaction time, the ring opening was performed under microwave irradiation $(200 \mathrm{~W})$ and solventless conditions, but no improvement of the yield of $\mathbf{7 a b}$ was observed (Table 2, entry 5). When using $n$-butylamine as 
nucleophile, the best yield of the corresponding sulfonyl $\beta$-amino alcohol 7ac was observed under solventless conditions at $78^{\circ} \mathrm{C}$ (Table 2, compare entries 5 and 6). No ring-opening reaction was observed with carbon nucleophiles such as sodium alkylmalonates, sodium cyanide, or trimethylsilyl cyanide under different conditions, only starting material being recovered from the reaction. Very recently, a highly regioselective ring opening of epoxides and aziridines using (bromodimethyl)sulfonium bromide has been reported. ${ }^{16}$ The ring opening of $\mathbf{6 a}$ with this reagent in MeCN at RT, led to the corresponding bromohydrin 7ad in a 69\% yield (Table 2, entry 7).

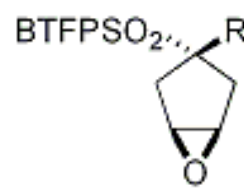

6

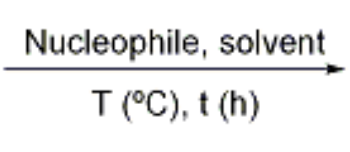

$\mathrm{T}\left({ }^{\circ} \mathrm{C}\right), \mathrm{t}(\mathrm{h})$

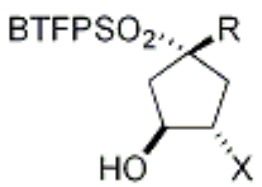

7

Scheme 4. Nucleophilic epoxide opening reactions.

At this point, the relative stereochemistry of epoxides $\mathbf{5}$ and $\mathbf{6}$ was confirmed by carrying out NOE studies on the ring-opening adducts $\mathbf{7}$ and $\mathbf{8}$. For this purpose, the diastereomeric epoxide $\mathbf{5 a}$ was also submitted to nucleophilic ring opening with $\mathrm{LiN}_{3}$ and $\mathrm{Me}_{2} \mathrm{SBr}_{2}$, to afford the alcohols 8aa and $8 \mathbf{a d}$ in a 62 and 67\% yield, respectively (Scheme 5, Table 1, entries 8 and 9). As depicted in Figure 3, the relative stereochemistry assigned to compounds $\mathbf{5}$ and $\mathbf{6}$ was corroborated by using the NOEs observed for the corresponding $\beta$-azido- alcohol compounds 7aa and 8aa as well as the bromohydrins 7ad and 8ad.

The BTFP sulfonyl epoxides $\mathbf{6 b}-\mathbf{e}$ were also submitted to ring opening with some of the previously studied nucleophiles (Table 1, entries 10-16). The use of (bromodimethyl)sulfonium bromide led to the $\beta$-bromo alcohols $\mathbf{7 b d}$, 7cd, 7dd, and 7ed with yields ranging from 54 to $87 \%$ (Table 1, entries 10-13). The BTFP sulfone 6d reacted in a very low isolated yield with benzylamine under solventless conditions at $80^{\circ} \mathrm{C}$ to give the functionalized sulfone $7 \mathbf{d d b}$ (Table 2, entry 14). Only a $34 \%$ yield of compound $\mathbf{7 c a}$ was obtained when BTFP-sulfone $\mathbf{6 c}$ reacted with $\mathrm{LiN}_{3}$ in DMF at $80^{\circ} \mathrm{C}$ for $24 \mathrm{~h}$ (Table 1, entry 15). This was due in part to the formation of compound 9 in a 31\% yield as a consequence of the hydrolysis and thermal decarboxylation of the isopropyl ester moiety (Figure 4). In order to avoid the hydrolysis of the ester group, the reaction was carried out in anhydrous conditions using $\mathrm{NaN}_{3}$ as nucleophile (Table 1, entry 16). However, under these conditions the carbonate $\mathbf{1 0}$ was isolated in a $52 \%$ yield as a result of the intramolecular nucleophilic attack of the alkoxide on the ester group (Figure 4).

Table 2. Nucleophilic epoxide-opening reactions

\begin{tabular}{|c|c|c|c|c|c|c|c|c|c|}
\hline \multirow[b]{2}{*}{ Entry } & \multicolumn{2}{|c|}{ Epoxide } & \multirow[b]{2}{*}{ Nu (equiv.) } & \multirow[b]{2}{*}{ Solvent } & \multirow[b]{2}{*}{$\mathrm{T}\left({ }^{\circ} \mathrm{C}\right)$} & \multirow[b]{2}{*}{$\mathrm{t}(\mathrm{h})$} & \multicolumn{3}{|c|}{ Product } \\
\hline & No. & $\mathrm{R}$ & & & & & No. & $\mathrm{X}$ & Yield $\%^{a}$ \\
\hline 1 & 6a & $\mathrm{Ph}$ & $\mathrm{NaN}_{3}(10)$ & DMF & 100 & 24 & $7 \mathbf{a a}$ & $\mathrm{N}_{3}$ & 54 \\
\hline 2 & 6a & $\mathrm{Ph}$ & $\operatorname{LiN}_{3}(10)$ & DMF & 80 & 24 & $7 \mathbf{a a}$ & $\mathrm{N}_{3}$ & 66 \\
\hline 3 & 6a & $\mathrm{Ph}$ & $\mathrm{BnNH}_{2}(20)$ & DMF & 100 & 24 & $7 \mathbf{a b}$ & $\mathrm{BnNH}$ & 20 \\
\hline 4 & $6 a$ & $\mathrm{Ph}$ & $\mathrm{BnNH}_{2}(30)$ & - & 80 & 70 & $7 a b$ & $\mathrm{BnNH}$ & 72 \\
\hline
\end{tabular}


Table 2. Continued

\begin{tabular}{|c|c|c|c|c|c|c|c|c|c|}
\hline \multirow[b]{2}{*}{ Entry } & \multicolumn{2}{|c|}{ Epoxide } & \multirow[b]{2}{*}{ Nu (equiv.) } & \multirow[b]{2}{*}{ Solvent } & \multirow[b]{2}{*}{$\mathrm{T}\left({ }^{\circ} \mathrm{C}\right)$} & \multirow[b]{2}{*}{$\mathrm{t}(\mathrm{h})$} & \multicolumn{3}{|c|}{ Product } \\
\hline & No. & $\mathrm{R}$ & & & & & No. & $\mathrm{X}$ & Yield $\%^{a}$ \\
\hline 5 & 6a & $\mathrm{Ph}$ & $\mathrm{BnNH}_{2}(5)$ & - & $95^{b}$ & 0.5 & $7 a b$ & $\mathrm{BnNH}$ & 40 \\
\hline 6 & 6a & $\mathrm{Ph}$ & $\mathrm{BuNH}_{2}(30)$ & - & 78 & 38 & $7 a c$ & BuNH & 63 \\
\hline 7 & $\mathbf{6 a}$ & $\mathrm{Ph}$ & $\mathrm{Me}_{2} \mathrm{SBr}_{2}(5)$ & $\mathrm{MeCN}$ & RT & 13 & 7ad & $\mathrm{Br}$ & 69 \\
\hline 8 & $5 a$ & $\mathrm{Ph}$ & $\operatorname{LiN}_{3}(10)$ & DMF & 80 & 24 & 8aa & $\mathrm{N}_{3}$ & 62 \\
\hline 9 & $5 a$ & $\mathrm{Ph}$ & $\mathrm{Me}_{2} \mathrm{SBr}_{2}(5)$ & $\mathrm{MeCN}$ & RT & 13 & 8ad & $\mathrm{Br}$ & 67 \\
\hline 10 & $6 \mathbf{b}$ & $4-\mathrm{NO}_{2} \mathrm{C}_{6} \mathrm{H}_{4}$ & $\mathrm{Me}_{2} \mathrm{SBr}_{2}(5)$ & $\mathrm{MeCN}$ & RT & 13 & 7bd & $\mathrm{Br}$ & 87 \\
\hline 11 & $6 c$ & $\mathrm{CO}_{2} \mathrm{Pr}^{i}$ & $\mathrm{Me}_{2} \mathrm{SBr}_{2}(5)$ & $\mathrm{MeCN}$ & RT & 13 & $7 \mathrm{~cd}$ & $\mathrm{Br}$ & 66 \\
\hline 12 & 6d & $\mathrm{CN}$ & $\mathrm{Me}_{2} \mathrm{SBr}_{2}(5)$ & $\mathrm{MeCN}$ & RT & 13 & 7dd & $\mathrm{Br}$ & 73 \\
\hline 13 & $6 e$ & $\mathrm{PO}(\mathrm{OEt})_{2}$ & $\mathrm{Me}_{2} \mathrm{SBr}_{2}(5)$ & $\mathrm{MeCN}$ & RT & 13 & 7ed & $\mathrm{Br}$ & 54 \\
\hline 14 & 6d & $\mathrm{CN}$ & $\mathrm{BnNH}_{2}(30)$ & - & 80 & 70 & $7 \mathrm{db}$ & $\mathrm{BnNH}$ & 15 \\
\hline 15 & 6c & $\mathrm{CO}_{2} \mathrm{Pr}^{i}$ & $\operatorname{LiN}_{3}(10)$ & DMF & 80 & 24 & $7 \mathrm{ca}$ & $\mathrm{N}_{3}$ & $34^{c}$ \\
\hline 16 & $6 c$ & $\mathrm{CO}_{2} \mathrm{Pr}^{i}$ & $\mathrm{NaN}_{3}(10)$ & DMF & 80 & 48 & 10 & $\mathrm{~N}_{3}$ & 52 \\
\hline
\end{tabular}

${ }^{a}$ Isolated yield after flash chromatography, based on starting epoxides. ${ }^{b}$ The reaction was performed under 200W microwave irradiation. ${ }^{c}$ A 31\% yield of 9 was also obtained.

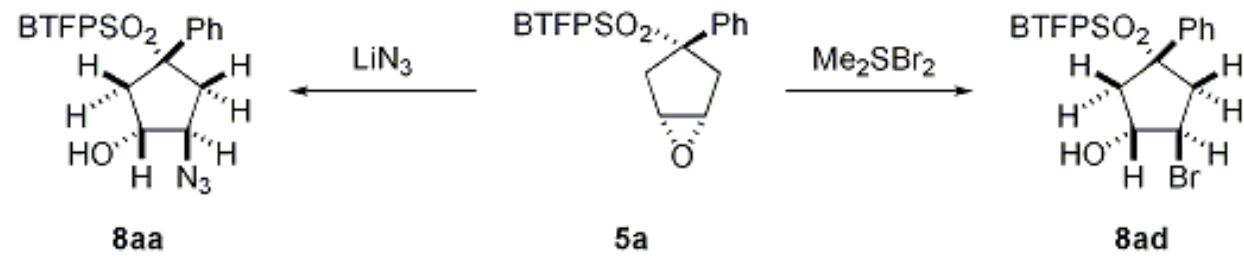

Scheme 5. Nucleophilic ring opening of epoxide 5a.

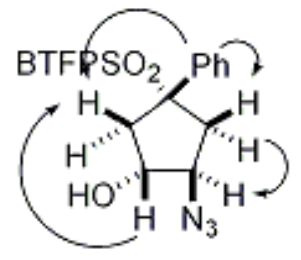

8 aa

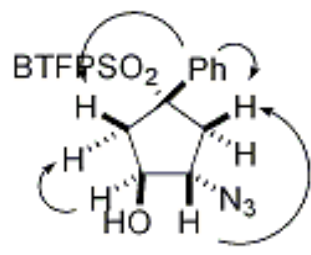

7 aa

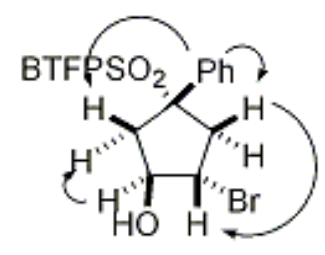

$7 \mathrm{ad}$

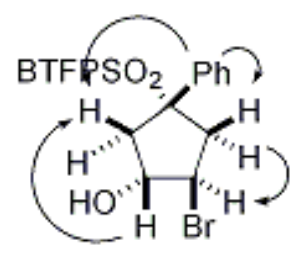

8ad

Figure 3. Determination of relative stereochemistry of compounds $\mathbf{7}$ and $\mathbf{8}$.

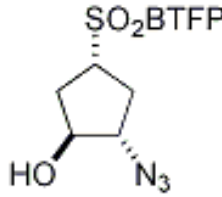

9

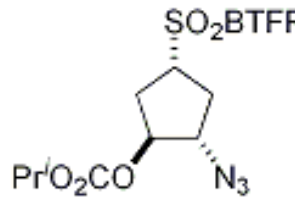

10

Figure 4. Azide ring opening reactions of $\mathbf{6 c}$. 
The last step of the synthesis of the 3,5-disubstituted cyclopentenones consisted of the oxidation and subsequent BTFP-sulfinic acid elimination from the cyclopentanols 7. The bromohydrin 7ad was chosen as a model substrate to perform an optimization of the reaction conditions since the obtained 5-bromocyclopentenone is a suitable substrate for further modification of the cyclopentene structure. As illustrated in Scheme 6, oxidation of the bromo cyclopentanol 7ad under Swern conditions (Method A) led, after in situ triethylamine (TEA)-catalyzed sulfinate elimination, to the corresponding 5-bromo-3-phenylcyclopent-2-enone (11) in 40\% yield. A similar result was obtained when the oxidation was carried out employing the system tetra- $n$-propylammonium perruthenate/ $N$-methylmorpholine- $N$-oxide $(\mathrm{TPAP} / \mathrm{NMO})^{17,18}$ where the in situ- generated $N$ methylmorpholine performed the elimination of the BTFP sulfinate (Method B, Scheme 6). A better (53\%) yield was obtained when the oxidation was carried out employing 2-iodoxybenzoic acid $(\mathrm{IBX})^{19}$ under base-free conditions (Method C). The low to moderate yields obtained in the final step of the cyclopentenone synthesis were probably associated with the isolation process, owing to the high volatility of the cyclopentenone 11, since the conversions in the oxidation reactions were in all cases very high. A similar study of reaction conditions was performed with the BTFP sulfones 7aa, 7bd, and 7ca, the best results being those depicted in Scheme 6. Methods A or B gave the best results for the oxidation-elimination sequence of the 2-azidocyclopentanol 7aa which afforded the cyclopent-2-enone 12 in a moderate 40\% yield. On the other hand, the cyclopentenones 13 and 14 were obtained after oxidation with IBX of 7bd and 7ca in 52 and 41\% yields, respectively (Scheme 5). The amino- alcohol derivatives such as $7 \mathbf{a b}$ and $7 \mathbf{a c}$ were also submitted to the oxidationelimination sequence under the studied conditions, but unfortunately only decomposition products were detected in the crude reaction mixtures.

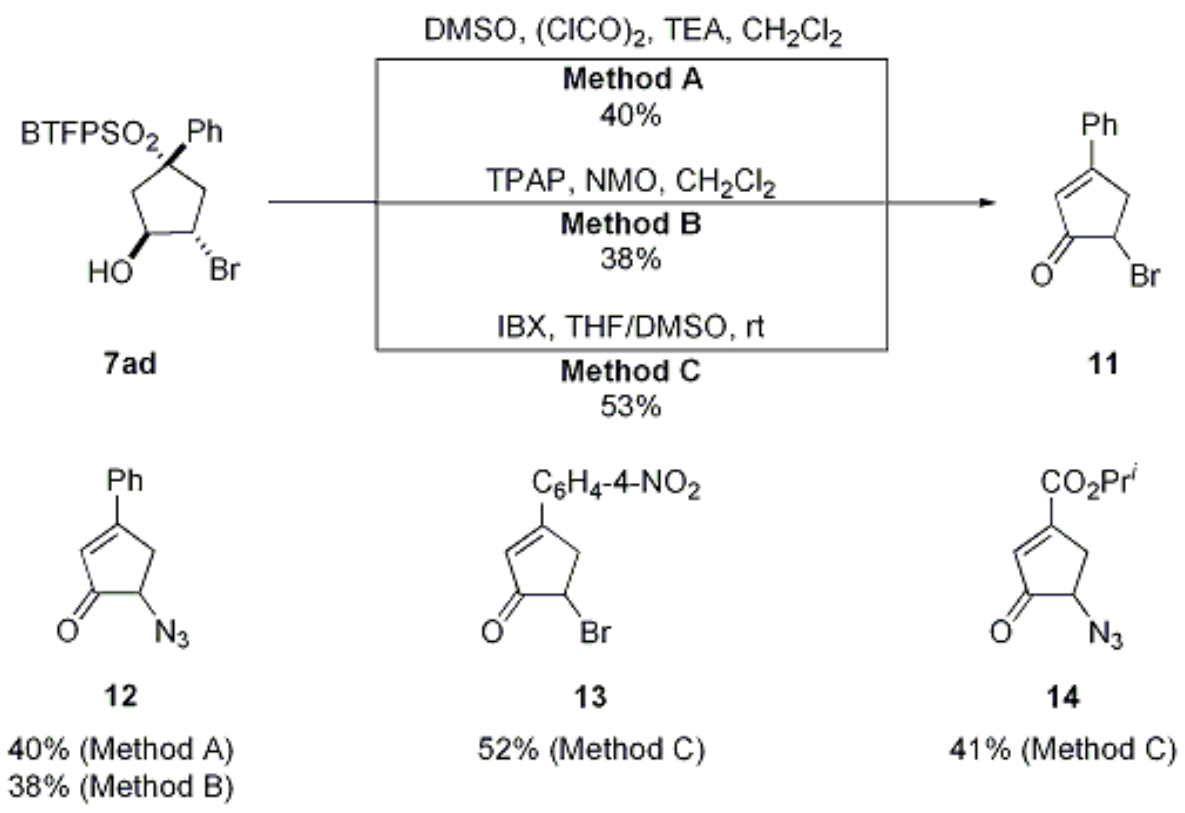

Scheme 6. Synthesis of 3,5-disubstituted cyclopentenones.

Finally, it is worth mentioning that the isolated total yield from the cyclopentenone synthesis can be improved if the synthetic sequence is performed without any purification and/or isolation of 
the corresponding intermediates. For example, the cyclopentenone $\mathbf{1 1}$ was obtained in a $40 \%$ isolated overall yield from BTFP sulfonyl cyclopentene 3a without any purification and/or separation of the intermediates $\mathbf{5 a / 6 a}$ and $\mathbf{7 a d}$ (Scheme 7). This result represents an increase of nearly $10 \%$ in yield compared to the previously described procedures.

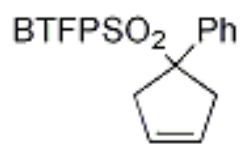

$3 a$

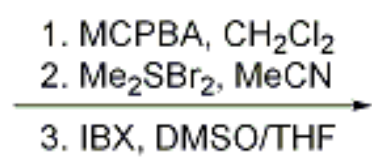

3. IBX, DMSO/THF

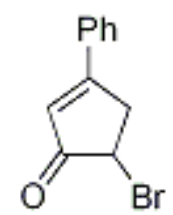

$11(40 \%)$

Scheme 7. Synthesis of cyclopentenone 11 without intermediate purifications.

In conclusion, this work further demonstrates the chemical versatility of BTFP sulfones, which can be used for the synthesis of highly functionalized 3,5-disubstituted cyclopent-2-enones. Owing to the strongly electron-withdrawing character of the BTFP sulfonyl group the dialkylation can be performed under milder conditions than previously reported (BuLi) for phenyl sulfones employing non-metallic bases such as $\mathrm{KOH}$ or $\mathrm{K}_{2} \mathrm{CO}_{3}$ under PTC conditions. The last oxidation-elimination step has been performed with different oxidants, such as Swern conditions, TPAP/NMO, and IBX. It can be concluded that IBX is the best reagent to perform this tandem process under very mild reaction conditions. The excellent nucleofugal properties of the BTFP sulfonyl group also facilitates the final elimination step. Consequently, the methodology described here makes use of readily available starting materials, mild reaction conditions, and very simple procedures, and is especially useful for the synthesis of new 5-bromocyclopentenone substrates, which allows future further functionalization.

\section{Experimental Section}

General Procedures. Melting points were obtained with a Reichert Thermovar apparatus and were not corrected. IR data were collected on a FTIR apparatus, Nicolet Impact 400D, and peaks are reported in $\mathrm{cm}^{-1}$. Only the structurally most important IR peaks have been listed. NMR spectra were recorded on a Bruker AC-300 (300 MHz for ${ }^{1} \mathrm{H}$ - NMR and $75 \mathrm{MHz}$ for ${ }^{13} \mathrm{C} \mathrm{NMR}$ ) using $\mathrm{CDCl}_{3}$ as solvent and TMS as internal standard unless otherwise noted; chemical shifts are given in $\delta(\mathrm{ppm})$ and coupling constants $(J)$ in Hz. Low-resolution electron impact (EI) mass spectra were obtained at $70 \mathrm{eV}$ on a Shimadzu QP-5000 and Agilent 5973 spectrometers, and fragment ions have $\mathrm{m} / z$ with relative intensities (\%) in parentheses. HRMS were performed on a Finnigan MAT 95S spectrometer. Analytical TLC was visualized with UV light at $254 \mathrm{~nm}$ or with $\mathrm{KMnO}_{4}$. Thin layer chromatography was carried out on TLC aluminum sheets with silica gel $60 \mathrm{~F}_{254}$ (Merck). For flash chromatography, silica gel $60(0.040-0.063 \mathrm{~mm})$ was employed. Microwave reactions were performed with a CEM Discover Synthesis Unit in glass vessels $(10 \mathrm{~mL})$ sealed with a septum under magnetic stirring. Reactions under inert atmosphere (argon) were performed in oven-dried 
glassware, sealed with a rubber septum, using anhydrous MeCN, THF or DMF. RT denotes room temperature.

Compounds 1a, ${ }^{11 \mathrm{c}} \mathbf{1 b},{ }^{11 \mathrm{c}} \mathbf{1 c},{ }^{8 \mathrm{a}, 8 \mathrm{~b}} \mathbf{2 a},{ }^{11 \mathrm{c}} \mathbf{2 b},{ }^{11 \mathrm{c}} \mathbf{2 c},{ }^{8 \mathrm{a}, 8 \mathrm{~b}} \mathbf{1 2}^{12}$ have been described previously.

General procedure for the synthesis of 3,5-bis-(trifluoromethyl)phenyl sulfanes 2 a-e. To a room temperature solution $\mathrm{NaH}(95 \%, 303 \mathrm{mg}, 12 \mathrm{mmol})$ in $\mathrm{MeCN}(60 \mathrm{~mL})$ under argon atmosphere, 3,5-bis-(trifluoromethyl)benzenethiol $(1.7 \mathrm{~mL}, 10 \mathrm{mmol}$ ) was added dropwise. After 20 min, the corresponding alkyl bromide $(11 \mathrm{mmol})$ was added and the reaction mixture was stirred at the same temperature for 1 day. After quenching with $\mathrm{H}_{2} \mathrm{O}(70 \mathrm{~mL})$, the mixture was extracted with EtOAc $(2 \times 50 \mathrm{~mL})$. The combined organic layers were dried $\left(\mathrm{MgSO}_{4}\right)$ and evaporated to afford the corresponding pure crude 3,5-bis-(trifluoromethyl)phenyl sulfanes 2a-e, which were used in the next oxidation step without further purification.

Cyanomethyl 3,5-bis-(trifluoromethyl)phenyl sulfane (2d). ${ }^{1} \mathrm{H}$ NMR $\delta 7.95$ (s, 2H, ArH), 7.87 (s, 1H, ArH), 3.71 (s, 2H, $\mathrm{CH}_{2} \mathrm{~S}$ ); ${ }^{13} \mathrm{C}$ NMR $\delta 135.3$ (ArC), 132.9 (q, $J_{\mathrm{C}-\mathrm{F}}=33.7,2 \times C_{3}$ ), 131.2 $(\mathrm{ArCH}), 122.6\left(\mathrm{q}, J_{\mathrm{C}-\mathrm{F}}=272.5,2 \times \mathrm{CF}_{3}\right), 122.5(\mathrm{ArCH}), 115.2(\mathrm{CN}), 20.5\left(\mathrm{CH}_{2}\right) ; \mathrm{MS} \mathrm{m} / z 286\left(\mathrm{M}^{+}+\right.$ 1, 11), 285 ( $\left.\mathrm{M}^{+}, 91\right), 266$ (21), 245 (100), 225 (46), 176 (14), 132 (10).

(Diethylphosphoryl)methyl 3,5-bis-(trifluoromethyl)phenyl sulfane (2e). ${ }^{1} \mathrm{H}$ NMR $\delta 7.84$ (s, $2 \mathrm{H}$, ArH), 7.69 (s, 1H, ArH), 4.17 (m, 4H, $\mathrm{OCH}_{2}$ ), 3.25 (d, $\left.J=13.6,2 \mathrm{H}, \mathrm{CH}_{2} \mathrm{~S}\right), 1.31$ (t, $J=7.1,6 \mathrm{H}, 2 \mathrm{x}$ $\left.\mathrm{CH}_{3}\right) ;{ }^{13} \mathrm{C}$ NMR $\delta 139.3(\mathrm{ArC}), 132.2\left(\mathrm{q}, J_{\mathrm{C}-\mathrm{F}}=34.0,2 \times \mathrm{CCF}_{3}\right), 128.3(\mathrm{ArCH}), 122.9\left(\mathrm{q}, J_{\mathrm{C}-\mathrm{F}}=\right.$ 273.3, 2 x CF CF $_{3} 120.1$ ( $\left.\mathrm{ArCH}\right), 63.0$ (d, $J_{\mathrm{C}-\mathrm{P}}=6.6,2$ x $\left.\mathrm{OCH}_{2}\right), 27.5$ (d, $\left.J_{\mathrm{C}-\mathrm{P}}=150.4,2 \times \mathrm{CH}_{2} \mathrm{~S}\right), 16.2$ (d, $\left.J_{\mathrm{C}-\mathrm{P}}=6.0,2 \times \mathrm{CH}_{3}\right)$; MS m/z $397\left(\mathrm{M}^{+}+1,10\right), 396\left(\mathrm{M}^{+}, 65\right), 377$ (14), 368 (18), 340 (18), 320 (19), 260 (23), 259 (100), 239 (46), 195 (13), 138 (13), 109 (25), 81 (14).

General procedure for the synthesis of 3,5-bis-(trifluoromethyl)phenyl sulfones 1a-c. To a RT stirred solution of the corresponding sulfide $\mathbf{2 a - c}(1 \mathrm{mmol})$ and $\mathrm{MnSO}_{4}$ monohydrate (2 mg, $1 \mathrm{~mol}$ \%) in MeCN (23 mL), was slowly added a previously prepared at $0{ }^{\circ} \mathrm{C}$ aqueous mixture comprised by $30 \% \mathrm{H}_{2} \mathrm{O}_{2}(5 \mathrm{mmol}, 515 \mu \mathrm{L})$ and a $0.2 \mathrm{M}$ buffer solution of $\mathrm{NaHCO}_{3}(17 \mathrm{~mL})$. After stirring for $1 \mathrm{~d}$ the reaction was quenched with a saturated aqueous solution of $\mathrm{NaCl}(30 \mathrm{~mL})$, extracted with EtOAc $(2 \times 20 \mathrm{~mL})$ and dried with anhydrous $\mathrm{Na}_{2} \mathrm{SO}_{4}$. Filtration and evaporation of the solvents afforded the corresponding pure crude sulfones $\mathbf{1 a - c}$ which were recrystallized in hexane.

General procedure for the synthesis of 3,5-bis-(trifluoromethyl)phenyl sulfones $1 \mathrm{~d}-\mathbf{e}$. To a stirred solution of the corresponding sulfide $\mathbf{2 d - e}(10 \mathrm{mmol})$ in a $1 / 1$ mixture of $\mathrm{MeOH} / \mathrm{H}_{2} \mathrm{O}(88$ $\mathrm{mL})$ at $0{ }^{\circ} \mathrm{C}$, was slowly added Oxone ${ }^{\circledR}(100 \mathrm{mmol}, 62 \mathrm{~g})$ and the resulting mixture was stirred at $\mathrm{RT}$ for $1 \mathrm{~d}$. Then, $\mathrm{MeOH}$ was evaporated, the residue was dissolved in $\mathrm{CH}_{2} \mathrm{Cl}_{2}(100 \mathrm{~mL})$ and filtered through Celite. To the resulting solution water was added and the mixture was extracted with $\mathrm{CH}_{2} \mathrm{Cl}_{2}(2 \times 50 \mathrm{~mL})$, was washed with a saturated solution of $\mathrm{NaCl}(3 \times 100 \mathrm{~mL})$, and dried $\left(\mathrm{MgSO}_{4}\right)$. Filtration and evaporation of the solvent afforded the corresponding pure crude sulfones 1d-e which were recrystallized in ether/hexane or purified by flash chromatography (hexane/EtOAc).

Cyanomethyl 3,5-bis-(trifluoromethyl)phenyl sulfone (1d). Beige solid; $\mathrm{R}_{f}$ (hexane/EtOAc: 4/1) 0.41; mp 114-116 ${ }^{\circ} \mathrm{C}$; IR v 3103, 3090, 2989, 2930, 2270, 1874, 1851, 1633, 1610, 1364, 1291, 1137; ${ }^{1} \mathrm{H}$ NMR $\delta 8.49$ (s, 2H, ArH), 8.29 (s, $\left.1 \mathrm{H}, \mathrm{ArH}\right), 4.18$ (s, 2H, $\left.\mathrm{CH}_{2} \mathrm{~S}\right) ;{ }^{13} \mathrm{C}$ NMR $(100 \mathrm{MHz}) \delta$ 139.1 (ArC), 136.9 (q, $\left.J_{\mathrm{C}-\mathrm{F}}=35.0,2 \times \mathrm{CCF}_{3}\right), 129.4,129.1(\mathrm{ArCH}), 122.0$ (q, $J_{\mathrm{C}-\mathrm{F}}=273.5,2 \mathrm{x}$ $\mathrm{CF}_{3}$ ), $109.5(\mathrm{CN}), 45.7\left(\mathrm{CH}_{2}\right)$; MS m/z 317 (M+, 0.5), 298 (11), 277 (44), 213 (100), 163 (14); HRMS Calcd for $\mathrm{C}_{10} \mathrm{H}_{5} \mathrm{~F}_{6} \mathrm{NO}_{2} \mathrm{~S}\left(\mathrm{M}^{+}\right)$316.9945, found: 316.9902. 
(Diethylphosphoryl)methyl 3,5-bis-(trifluoromethyl)phenyl sulfone (1e). White solid; $\mathrm{R}_{f}$ (hexane/EtOAc: 2/1) 0.17; mp 78-80 ${ }^{\circ} \mathrm{C}$; IR $v$ 3090, 2985, 2908, 1633, 1337, 1278, 1159, 1137, 1014; ${ }^{1} \mathrm{H}$ NMR $\delta 8.48$ (s, $2 \mathrm{H}, \mathrm{ArH}$ ), 8.17 (s, $1 \mathrm{H}, \mathrm{ArH}$ ), 4.17 (sept., $J=7.4,4 \mathrm{H}, 2$ x $\mathrm{OCH}_{2}$ ), 3.84 (d, $J=16.7,2 \mathrm{H}, \mathrm{CH}_{2} \mathrm{~S}$ ), 1.29 (t, $\left.J=7.1,6 \mathrm{H}, 2 \times \mathrm{CH}_{3}\right) ;{ }^{13} \mathrm{C} \mathrm{NMR} \delta 142.1(\mathrm{ArC}), 132.8$ (q, $J_{\mathrm{C}-\mathrm{F}}=35.0,2$ x $\left.C \mathrm{CF}_{3}\right), 129.3,127.6(\mathrm{ArCH}), 122.3\left(\mathrm{q}, J_{\mathrm{C}-\mathrm{F}}=273.5,2 \times \mathrm{CF}_{3}\right), 63.6\left(\mathrm{~d}, J_{\mathrm{C}-\mathrm{P}}=6.7,2 \times \mathrm{OCH}_{2}\right), 53.9$ (d, $\left.J_{\mathrm{C}-\mathrm{P}}=137.4,2 \times \mathrm{CH}_{2} \mathrm{~S}\right), 16.1$ (d, $\left.J_{\mathrm{C}-\mathrm{P}}=5.4,2 \times \mathrm{CH}_{3}\right) ; \mathrm{MS} m / z 428\left(\mathrm{M}^{+}, 1\right), 409$ (29), 401 (66), 383 (33), 373 (46), 355 (39), 354 (11), 353 (94), 337 (21), 336 (30), 309 (38), 308 (47), 293 (12), 292 (43), 290 (18), 277 (29), 261 (44), 257 (12), 230 (43), 227 (100), 213 (95), 208 (28), 194 (28), 163 (28), 144 (23), 143 (15), 125 (30), 124 (22), 123 (24), 109 (38), 108 (43), 107 (29), 97 (78), 96 (29), 95 (37), 93 (33), 81 (28), 80 (41), 79 (47), 78 (24), 65 (44), 45 (13), 43 (13); HRMS Calcd for $\mathrm{C}_{13} \mathrm{H}_{15} \mathrm{~F}_{6} \mathrm{O}_{5} \mathrm{PS}\left(\mathrm{M}^{+}\right)$, 428.0282, found: 428.0281 .

General procedure for the synthesis of [3,5-bis-(trifluoromethyl)phenylsulfonyl]cyclopentenes, 3. To a $\mathrm{CH}_{3} \mathrm{CN}(40 \mathrm{~mL})$ solution of $\mathrm{TBAB}(65 \mathrm{mg}, 0.2 \mathrm{mmol})$ and the corresponding sulfone (2 mmol) at RT, was added $\mathrm{KOH}(1.08 \mathrm{~g}, 18 \mathrm{mmol}$, for sulfone 1a-b) or $\mathrm{K}_{2} \mathrm{CO}_{3}$ (1.656 g, $18 \mathrm{mmol}$, for sulfones 1c-e). The mixture was stirred for $30 \mathrm{~min}$ and cis-1,4dichloro-2-butene ( $240 \mu \mathrm{L}, 2.2 \mathrm{mmol}$ ) was added. The reaction mixture was stirred overnight and quenched with a saturated solution of $\mathrm{NH}_{4} \mathrm{Cl}(50 \mathrm{~mL})$, extracted with EtOAc $(2 \times 50 \mathrm{~mL})$, and the organic phase was dried $\left(\mathrm{MgSO}_{4}\right)$. Filtration and solvent evaporation afforded the corresponding crude cyclopentenes, which were purified by flash chromatography (hexane/EtOAc) to afford pure compounds 3 .

1-[3,5-Bis-(trifluoromethyl)phenylsulfonyl]-1-phenyl-3-cyclopentene (3a). White solid; $\mathbf{R}_{f}$ (hexane/EtOAc: 2/1) 0.68; mp 95-96 ${ }^{\circ} \mathrm{C}$; IR $v$ 3073, 3042, 2914, 2853, 1629, 1607, 1453, 1352, 1282, 1150; ${ }^{1} \mathrm{H}$ NMR $\delta 8.02$ (s, 1H, ArH), 7.75 (s, 2H, ArH), 739-7.27 (m, 3H, ArH), 7.19 (d, $J=$ 7.0, 2H, ArH), 5.75 (s, 2H, CH=CH), $3.62\left(\mathrm{~d}, J=16.4,2 \mathrm{H}, \mathrm{CH}_{2}\right), 3.15$ (d, $\left.J=16.7,2 \mathrm{H}, \mathrm{CH}_{2}\right) ;{ }^{13} \mathrm{C}$ NMR $\delta$ 138.2, $136.6(\mathrm{ArC}), 132.0$ (q, $J_{\mathrm{C}-\mathrm{F}}=34.4,2 \times C_{C F_{3}}$ ), 130.3, 129.9 129.1, 128.4, 126.8 $(\mathrm{ArCH}), 128.3(\mathrm{CH}=\mathrm{CH}), 122.2\left(\mathrm{q}, J_{\mathrm{C}-\mathrm{F}}=274.3,2 \times \mathrm{CF}_{3}\right), 77.6(\mathrm{CS}), 40.9\left(2 \times \mathrm{CH}_{2}\right) ; \mathrm{MS} m / z 401$ $\left(\mathrm{M}^{+}\right.$- F, 0.4), 144 (17), 143 (100), 142 (14), 141 (21), 128 (60), 115 (20); HRMS Calcd for $\mathrm{C}_{19} \mathrm{H}_{14} \mathrm{~F}_{6} \mathrm{O}_{2} \mathrm{~S}\left(\mathrm{M}^{+}\right)$420.0619, ( $\mathrm{M}^{+}$- F) 401.0635, found: 401.0681.

1-[3,5-Bis-(trifluoromethyl)phenylsulfonyl]-1-(4-nitrophenyl)-3-cyclopentene (3b). Yellow solid; $\mathrm{R}_{f}$ (hexane/EtOAc: 2/1) 0.63; mp 202-204 ${ }^{\circ} \mathrm{C}$; IR $v$ 3080, 2951, 2856, 1607, 1513, 1354, 1283, 1142; ${ }^{1} \mathrm{H}$ NMR $\delta$ 8.21, 7.50 (2d, $J=8.9,4 \mathrm{H}, \mathrm{ArH}$ ), 8.10 (s, $\left.1 \mathrm{H}, \mathrm{ArH}\right), 7.95$ (s, $\left.2 \mathrm{H}, \mathrm{ArH}\right), 5.61$ (s, $2 \mathrm{H}, \mathrm{CH}=\mathrm{CH}$ ), 3.63 (d, $J=16.5,2 \mathrm{H}, \mathrm{CH}_{2}$ ), $3.16\left(\mathrm{~d}, J=16.7,2 \mathrm{H}, \mathrm{CH}_{2}\right) ;{ }^{13} \mathrm{C} \mathrm{NMR} \delta 148.0,143.0$, 138.3 (ArC), 132.6 (q, $\left.J_{\mathrm{C}-\mathrm{F}}=35.1,2 \times C C_{3}\right), 131.32,130.3$ 128.3, $123.3(\mathrm{CH}=\mathrm{CH}, \mathrm{ArCH}), 122.1$ $\left(\mathrm{q}, J_{\mathrm{C}-\mathrm{F}}=274.3,2 \times \mathrm{CF}_{3}\right), 77.7(\mathrm{CS}), 41.2\left(2 \times \mathrm{CH}_{2}\right) ; \mathrm{MS} \mathrm{m} / z 188\left(\mathrm{M}^{+}-\mathrm{BTFPSO}_{2}, 100\right), 142(49)$, 141 (49), 139 (10), 128 (11), 115 (34); HRMS Calcd for $\mathrm{C}_{19} \mathrm{H}_{13} \mathrm{~F}_{6} \mathrm{NO}_{4} \mathrm{~S}\left(\mathrm{M}^{+}\right)$465.0469, $\left(\mathrm{M}^{+}-\mathrm{F}\right)$ 446.0485, found: 446.0488 .

Isopropyl 1-[3,5-bis-(trifluoromethyl)phenylsulfonyl]-3-cyclopentenecarboxylate (3c). White solid; $\mathrm{R}_{f}$ (hexane/EtOAc: 9/1) 0.55; mp 90-92 ${ }^{\circ} \mathrm{C}$; IR $v$ 3107, 2980, 2932, 1718, 1447, 1363, 1333, 1297, 1141; ${ }^{1} \mathrm{H}$ NMR $\delta 8.33$ (s, 2H, ArH), 8.16, (s, $1 \mathrm{H}, \mathrm{ArH}$ ), 5.67 (s, 2H, CH=CH), 4.98 (sept., $J=$ 6.2, $1 \mathrm{H}, \mathrm{OCH}), 3.21\left(2 \mathrm{~d}, J=16.4,4 \mathrm{H}, 2 \times \mathrm{CH}_{2}\right), 1.23$ (d, $\left.J=6.2,6 \mathrm{H}, 2 \times \mathrm{CH}_{3}\right) ;{ }^{13} \mathrm{C}$ NMR $\delta 167.4$ $\left(\mathrm{CO}_{2} \mathrm{Et}\right), 139.7(\mathrm{ArC}), 132.5\left(\mathrm{q}, J_{\mathrm{C}-\mathrm{F}}=34.0,2 \times \mathrm{CCF}_{3}\right), 130.4,127.5(\mathrm{ArCH}), 127.4(\mathrm{CH}=\mathrm{CH})$, $122.3\left(\mathrm{q}, J_{\mathrm{C}-\mathrm{F}}=273.5,2 \times \mathrm{CF}_{3}\right), 78.1(\mathrm{CS}), 71.4(\mathrm{OCH}), 38.9\left(2 \times \mathrm{CH}_{2}\right), 21.2\left(2 \times \mathrm{CH}_{3}\right) ; \mathrm{MS} \mathrm{m} / \mathrm{z}$ 430 (M+ $\left.\mathrm{M}^{+}, 0.3\right), 257$ (16), 239 (11), 239 (177), 150 (13), 149 (100), 129 (12), 125 (11), 111 (26), 105 (14), 99 (10), 98 (11), 97 (26), 95 (12), 93 (12), 85 (26), 84 (14), 83 (25), 82 (11), 81 (16), 73 (17), 
71 (37), 69 (28), 67 (14), 60 (18), 57 (57), 56 (34), 55 (36), 44 (15), 43 (52), 41 (35); HRMS Calcd for $\mathrm{C}_{17} \mathrm{H}_{16} \mathrm{~F}_{6} \mathrm{O}_{4} \mathrm{~S}\left(\mathrm{M}^{+}\right)$430.0673, $\left(\mathrm{M}^{+}-\mathrm{OPr}^{\mathrm{i}}\right)$ 371.0177, found: 371.0197.

1-[3,5-Bis-(trifluoromethyl)phenylsulfonyl]-1-cyanocyclopent-3-ene (3d). White solid; $\mathrm{R}_{f}$ (hexane/EtOAc: 4/1) 0.54; mp 146-148 ${ }^{\circ} \mathrm{C}$; IR v 3080, 2926, 2862, 2238, 1628, 1442, 1364, 1332, 1282, 1200, 1132; ${ }^{1} \mathrm{H}$ NMR $\delta 8.50$ (s, 2H, ArH), 8.27 (s, 1H, ArH), 5.76 (s, 2H, CH=CH), 3.39 (d, $J$ $\left.=15.2,2 \mathrm{H}, \mathrm{CH}_{2}\right), 3.02\left(\mathrm{~d}, J=15.6,2 \mathrm{H}, \mathrm{CH}_{2}\right) ;{ }^{13} \mathrm{C}$ NMR $(100 \mathrm{MHz}) \delta 137.5(\mathrm{ArC}), 133.5\left(\mathrm{q}, J_{\mathrm{C}-\mathrm{F}}=\right.$ 35.0, $\left.2 \times C_{C F}\right), 130.6,128.8(\mathrm{ArCH}), 127.0(\mathrm{CH}=\mathrm{CH}), 122.1\left(\mathrm{q}, J_{\mathrm{C}-\mathrm{F}}=274.8,2 \times \mathrm{CF}_{3}\right), 118.1$ (CN), 64.7 (CCN), 41.0 (2 x CH ); MS m/z 369 (M+1, 0.1), 312 (11), 257 (38), 239 (19), 213 (15), 177 (13), 167 (15), 150 (13), 149 (100), 129 (15), 111 (11), 97 (16), 92 (36), 91 (45), 85 (15), 83 (18), 81 (12), 73 (18), 71 (27), 70 (14), 69 (22), 65 (16), 60 (11), 57 (45), 56 (44), 55 (28), 43 (46), 41 (26); HRMS Calcd for $\mathrm{C}_{14} \mathrm{H}_{9} \mathrm{~F}_{6} \mathrm{NO}_{2} \mathrm{~S}\left(\mathrm{M}^{+}\right)$369.0258, $\left(\mathrm{M}^{+}-\mathrm{F}\right) 350.0274$, found: 350.0262.

Diethyl 1-[3,5-bis-(trifluoromethyl)phenylsulfonyl]-3-cyclopentenylphosphonate (3e). White solid; $\mathrm{R}_{f}$ (hexane/EtOAc: 4/1) 0.13; mp 71-73 ${ }^{\circ} \mathrm{C}$; IR $v$ 3076, 2994, 2930, 2857, 1360, 1287, 1260, 1150, 1023; ${ }^{1} \mathrm{H}$ NMR $\delta 8.47$ (s, 2H, ArH), 8.14 (s, 1H, ArH), 5.63 (s, 2H, CH=CH), 4.21-4.12 (m, $\left.4 \mathrm{H}, 2 \times \mathrm{OCH}_{2}\right), 3.34-3.07\left(\mathrm{~m}, 4 \mathrm{H}, 2 \mathrm{x}=\mathrm{CHCH}_{2}\right), 1.27\left(\mathrm{t}, J=7.0,6 \mathrm{H}, 2 \times \mathrm{CH}_{3}\right) ;{ }^{13} \mathrm{C} \mathrm{NMR} \delta 140.3$ $(\mathrm{ArC}), 132.2\left(\mathrm{q}, J_{\mathrm{C}-\mathrm{F}}=34.0,2 \times C_{C F_{3}}\right), 131.5(\mathrm{CH}=\mathrm{CH}), 127.6,127.5(\mathrm{ArCH}), 122.4\left(\mathrm{q}, J_{\mathrm{C}-\mathrm{F}}=\right.$ 273.3, $2 \times \mathrm{CF}_{3}$ ), 70.3 (d, $\left.J_{\mathrm{C}-\mathrm{P}}=143.8, \mathrm{CP}\right), 63.8$ (d, $\left.J_{\mathrm{C}-\mathrm{P}}=6.6,2 \times \mathrm{OCH}_{2}\right), 38.7\left(2 \times \mathrm{CH}=\mathrm{CH}_{2}\right), 16.1$ (d, $J_{\mathrm{C}-\mathrm{P}}=5.5,2 \times \mathrm{CH}_{3}$ ); MS m/z 480 (M $\left.\mathrm{M}^{+}, 0.01\right), 204$ (10), 203 (100), 202 (23), 175 (19), 149 (33), 147 (61), 146 (18), 129 (20), 92 (10), 81 (13), 66 (25), 65 (21); HRMS Calcd for $\mathrm{C}_{17} \mathrm{H}_{19} \mathrm{~F}_{6} \mathrm{O}_{5} \mathrm{PS}$ $\left(\mathrm{M}^{+}\right)$480.0595, $\left(\mathrm{M}^{+}-\mathrm{C}_{13} \mathrm{H}_{9} \mathrm{~F}_{6} \mathrm{O}_{2} \mathrm{~S}\right)$ 137.0368, found: 137.0378 .

1-(4-Nitrophenyl)-3-cyclopentenol (4). Yellow oil; $\mathrm{R}_{f}$ (hexane/EtOAc: 4/1) 0.15; IR $v$ 3424, 3073, 2923, 2853, 1601, 1521, 1335; ${ }^{1} \mathrm{H}$ NMR $\delta$ 8.17, 7.62 (2d, $\left.J=8.9,2 \mathrm{H}, \mathrm{ArH}\right), 5.83$ (s, 2H, CH=CH), 2.94, 2.77 (2d, $J=16.2,2 \mathrm{H}, \mathrm{CH}_{2}$ ); ${ }^{13} \mathrm{C}$ NMR $\delta$ 154.6, 146.6 (ArC), 128.2, 125.7, 123.3 (ArCH, $\mathrm{CH}=\mathrm{CH}), 81.7$ (COH), 51.0 (2 x CH ); MS m/z 205 (M+1 10), 151 (11), 150 (100), 104 (12); HRMS Calcd for $\mathrm{C}_{11} \mathrm{H}_{11} \mathrm{NO}_{3}\left(\mathrm{M}^{+}\right)$205.0739, found: 205.0719.

General procedure for the synthesis of [3,5-bis-(trifluoromethyl)phenyl]sulfonyl oxiranes, 5 and 6. To a solution of [3,5-bis-(trifluoromethyl)phenylsulfonyl]cyclopentene, 3 (5 mmol), in $\mathrm{CH}_{2} \mathrm{Cl}_{2}(100 \mathrm{~mL})$ at RT, MCPBA $(6.75 \mathrm{~g}, 30 \mathrm{mmol})$ was added and the mixture was heated at $50{ }^{\circ} \mathrm{C}$ for $18 \mathrm{~h}$. The reaction mixture was then quenched with a saturated solution of $\mathrm{NaHCO}_{3}(100 \mathrm{~mL})$, and extracted with $\mathrm{CH}_{2} \mathrm{Cl}_{2}(2 \times 80 \mathrm{~mL})$. The organic phase was further washed with a saturated solution of $\mathrm{Na}_{2} \mathrm{SO}_{3}(3 \times 50 \mathrm{~mL})$ and $\mathrm{NaHCO}_{3}(2 \times 50 \mathrm{~mL})$. The organic phase was dried $\left(\mathrm{MgSO}_{4}\right)$, and after solvent evaporation the corresponding crude oxiranes were obtained as a mixture of diastereomers (see Scheme 3), which were purified by flash chromatography (hexane/EtOAc) to afford pure compounds 5 and 6.

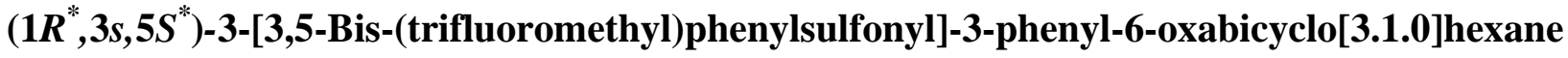

(6a). White solid; $\mathrm{R}_{f}$ (hexane/EtOAc: 2/1) 0.64; mp 153-155 ${ }^{\circ} \mathrm{C}$; IR $v$ 3076, 3038, 2940, 1623, 1443, 1355, 1290, 1132; ${ }^{1} \mathrm{H}$ NMR $\delta 8.00$ (s, 1H, ArH), 7.54 (s, 2H, ArH), 7.32-7.20 (m, 3H, ArH), 7.10 (d, $J=7.6,2 \mathrm{H}, \mathrm{ArH}), 3.72$ (s, $2 \mathrm{H}, 2 \mathrm{x} \mathrm{OCH}), 3.04,2.82\left(2 \mathrm{~d}, J=15,4 \mathrm{H}, 2 \times \mathrm{CH}_{2}\right) ;{ }^{13} \mathrm{C} \mathrm{NMR} \delta$ 138.0, 136.0 (ArC), 131.9 (q, $\left.J_{\mathrm{C}-\mathrm{F}}=35.0,2 \times C_{C F_{3}}\right), 129.9,128.8,128.6,128.2,126.9(\mathrm{ArCH})$, $122.1\left(\mathrm{q}, J_{\mathrm{C}-\mathrm{F}}=273.5,2 \times \mathrm{CF}_{3}\right), 76.8(\mathrm{CS}), 56.9(2 \times \mathrm{OCH}), 35.3\left(2 \times \mathrm{CH}_{2}\right) ; \mathrm{MS} \mathrm{m} / \mathrm{z}\left(\mathrm{M}^{+}-\right.$ BTFPSO $\left._{2}, 100\right), 144$ (15), 141 (22), 131 (35), 129 (30), 128 (17), 116 (17), 115 (53), 103 (12), 91 (74), 77 (14), 43 (11); HRMS Calcd for $\mathrm{C}_{19} \mathrm{H}_{14} \mathrm{~F}_{6} \mathrm{O}_{3} \mathrm{~S}\left(\mathrm{M}^{+}\right)$436.0568, $\left(\mathrm{M}^{+}-\mathrm{F}\right)$ 417.0584, found: 417.0597. 
$\left(1 R^{*}, 3 r, 5 S^{*}\right)-3$-[3,5-Bis-(trifluoromethyl)phenylsulfonyl]-3-phenyl-6-oxabicyclo[3.1.0]hexane (5a). White solid; $\mathrm{R}_{f}$ (hexane/EtOAc: 2/1) 0.26; mp 168-170 ${ }^{\circ} \mathrm{C}$; IR $v$ 3089, 3064, 3050, 2937, 1626, 1361, 1272, 1125; ${ }^{1} \mathrm{H}$ NMR $\delta 7.95$ (s, $\left.1 \mathrm{H}, \mathrm{ArH}\right), 7.83$ (s, 2H, ArH), 7.28-7.20 (m, 3H, ArH), 7.14 (d, $J=7.5,2 \mathrm{H}, \mathrm{ArH}), 3.62$ (s, $2 \mathrm{H}, 2 \mathrm{x} \mathrm{OCH}), 3.40,2.65$ (2d, $\left.J=16,4 \mathrm{H}, 2 \times \mathrm{CH}_{2}\right) ;{ }^{13} \mathrm{C} \mathrm{NMR}$ $(100 \mathrm{MHz}) \delta 140.6,138.1$ (ArC), 131.9 (q, $\left.J_{\mathrm{C}-\mathrm{F}}=34.1,2 \times C C_{3}\right), 129.9,129.5,129.0,128.4,126.4$ $(\mathrm{ArCH}), 122.3\left(\mathrm{q}, J_{\mathrm{C}-\mathrm{F}}=273.3,2 \times \mathrm{CF}_{3}\right), 78.7(\mathrm{CS}), 56.2(2 \times \mathrm{OCH}), 37.2\left(2 \times \mathrm{CH}_{2}\right) ; \mathrm{MS} \mathrm{m} / z 436$ ( $\left.\mathrm{M}^{+}, 0.01\right), 160$ (11), 159 (100), 144 (10), 141 (14), 131 (22), 129 (18), 128 (11), 116 (11), 115 (32), 91 (42); HRMS Calcd for $\mathrm{C}_{19} \mathrm{H}_{14} \mathrm{~F}_{6} \mathrm{O}_{3} \mathrm{~S}\left(\mathrm{M}^{+}\right)$436.0568, ( $\left.\mathrm{M}^{+}-\mathrm{F}\right)$ 417.0584, found: 417.0566. $\left(1 R^{*}, 3 s, 5 S^{*}\right)$-3-[3,5-Bis-(trifluoromethyl)phenylsulfonyl]-3-(4-nitrophenyl)-6-oxabicyclo[3.1.0]hexane (6b). White solid; $\mathrm{R}_{f}$ (pentane/EtOAc: 6/1) 0.15; mp 225-227 ${ }^{\circ} \mathrm{C}$; IR $v$ 3089, 3035, 2937, 1596, 1523, 1351, 1277, 1149; ${ }^{1} \mathrm{H}$ NMR $\delta$ 8.12, 7.37 (2d, $\left.J=8.9,4 \mathrm{H}, \operatorname{ArH}\right), 8.05$ (s, $1 \mathrm{H}$, $\mathrm{ArH}), 7.59$ (s, $2 \mathrm{H}, \mathrm{ArH}), 3.75$ (s, 2H, 2 x OCH), 3.09, 2.89 (2d, $\left.J=14.8,2 \mathrm{H}, \mathrm{CH}_{2}\right) ;{ }^{13} \mathrm{C}$ NMR $\delta$ 147.7, 143.3, 137.8 (ArC), 132.7 (q, $\left.J=35.0,2 \times C C_{3}\right), 129.6,127.5,123.2(\mathrm{ArH}), 122.5\left(\mathrm{q}, J_{\mathrm{C}-\mathrm{F}}=\right.$

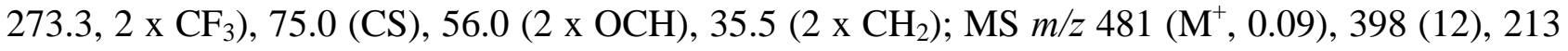
(18), 205 (26), 204 (100), 187 (16), 160 (13), 158 (45), 157 (14), 136 (32), 131 (10), 130 (29), 129 (53), 128 (51), 127 (19), 116 (14), 115 (39), 102 (12); HRMS Calcd for $\mathrm{C}_{19} \mathrm{H}_{13} \mathrm{~F}_{6} \mathrm{NO}_{5} \mathrm{~S}\left(\mathrm{M}^{+}\right.$) 481.0419, found: 481.0425 .

$\left(1 R^{*}, 3 s, 5 S^{*}\right)$-Isopropyl 3-[3,5-bis-(trifluoromethyl)phenylsulfonyl]-6-oxabicyclo-[3.1.0]-hexane 3-carboxylate (6c). White solid; $\mathrm{R}_{f}$ (hexane/EtOAc: 2/1) 0.21; mp $115-117{ }^{\circ} \mathrm{C}$; IR $v 3091$, 3055, 2983, 1729, 1611, 1363, 1275, 1131; ${ }^{1} \mathrm{H}$ NMR $\delta 8.21$ (s, 2H, ArH), 8.18 (s, $\left.1 \mathrm{H}, \mathrm{ArH}\right), 4.89$ [sept., $J$ $\left.=6.2,1 \mathrm{H}, \mathrm{CH}\left(\mathrm{CH}_{3}\right)_{2}\right], 3.62(\mathrm{~s}, 2 \mathrm{H}, 2 \times \mathrm{OCH}), 3.02,2.54\left(2 \mathrm{~d}, J=14,4 \mathrm{H}, 2 \times \mathrm{CH}_{2}\right), 1.19$ (d, $J=6.2$, 6H, $\left.2 \times \mathrm{CH}_{3}\right) ;{ }^{13} \mathrm{C}$ NMR $\delta 166.3(\mathrm{CO}), 139.4(\mathrm{ArC}), 132.8$ (q, $\left.J_{\mathrm{C}-\mathrm{F}}=35.1,2 \times \mathrm{CCF}_{3}\right), 130.0,127.8$ $(\mathrm{ArCH}), 122.3\left(\mathrm{q}, J_{\mathrm{C}-\mathrm{F}}=273.3,2 \times \mathrm{CF}_{3}\right), 74.3(\mathrm{CS}), 71.3\left[\mathrm{OCH}\left(\mathrm{CH}_{3}\right)_{2}\right], 54.7(2 \mathrm{x} \mathrm{OCH}), 33.6(2 \mathrm{x}$ $\mathrm{CH}_{2}$ ), 21.1 (2 x CH ); MS m/z 446 (M+ 0.05), 261 (16), 177 (24), 150 (12), 149 (100), 127 (11), 126 (36), 125 (17), 99 (11), 97 (13), 83 (10), 81 (15), 43 (28), 41 (11); HRMS Calcd for $\mathrm{C}_{17} \mathrm{H}_{16} \mathrm{~F}_{6} \mathrm{O}_{5} \mathrm{~S}\left(\mathrm{M}^{+}\right)$446.0623, ( $\mathrm{M}^{+}$- F) 427.0639, found: 427.0667.

$\left(1 R^{*}, 3 s, 5 S^{*}\right)$-3-[3,5-Bis-(trifluoromethyl)phenylsulfonyl]-3-cyano-6-oxabicyclo[3.1.0]hexane

(6d). White solid; $\mathrm{R}_{f}$ (hexane/EtOAc: $2 / 1$ ) 0.45; mp 164-166 ${ }^{\circ} \mathrm{C}$; IR $v$ 3113, 3059, 3025, 2956, 2244, 1631, 1351, 1282, 1140; ${ }^{1} \mathrm{H}$ NMR $\delta 8.43$ (s, 2H, ArH), 8.28 (s, 1H, ArH), 3.78 (s, 2H, 2 x $\mathrm{OCH}$ ), 2.72 (d, $\left.J=14.4,2 \mathrm{H}, \mathrm{CH}_{2}\right), 2.64$ (d, $\left.J=14.3,2 \mathrm{H}, \mathrm{CH}_{2}\right) ;{ }^{13} \mathrm{C}$ NMR $(100 \mathrm{MHz}) \delta 137.5$ $(\operatorname{ArC}), 133.6\left(\mathrm{q}, J_{\mathrm{C}-\mathrm{F}}=35.0,2 \times \mathrm{CCF}_{3}\right), 130.5,129.0(\mathrm{ArCH}), 122.0\left(\mathrm{q}, J_{\mathrm{C}-\mathrm{F}}=273.5,2 \times \mathrm{CF}_{3}\right)$, 117.5 (CN), 61.9 (CS), 55.3 (2 x OCH), 35.3 (2 x CH 2 ); MS m/z 385 (M+ $\mathrm{M}^{+}$044), 366 (45), 276 (19), 262 (10), 261 (25), 214 (16), 213 (95), 195 (13), 194 (30), 163 (25), 144 (23), 143 (13), 108 (44), 107 (100), 106 (46), 91 (91), 81 (21), 80 (37), 79 (18), 53 (50), 52 (17); HRMS Calcd for $\mathrm{C}_{14} \mathrm{H}_{9} \mathrm{~F}_{6} \mathrm{NO}_{3} \mathrm{~S}\left(\mathrm{M}^{+}\right)$385.0207, $\left(\mathrm{M}^{+}-\mathrm{F}\right)$ 366.0223, found: 366.0229.

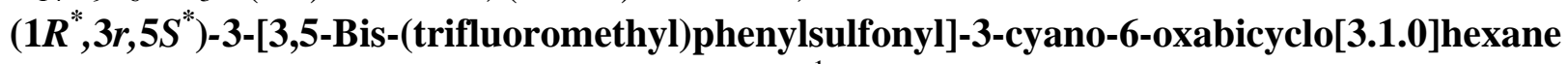

(5d). White solid; $\mathrm{R}_{f}$ (hexane/EtOAc: 2/1) 0.45; ${ }^{1} \mathrm{H}$ NMR $\delta 8.43$ (s, 2H, ArH), 8.27 (s, 1H, ArH), 3.70 (s, 2H, $2 \times \mathrm{OCH}$ ), 2.99 (d, $\left.J=16.0,2 \mathrm{H}, \mathrm{CH}_{2}\right), 2.61$ (d, $\left.J=15.1,2 \mathrm{H}, \mathrm{CH}_{2}\right) ;{ }^{13} \mathrm{C}$ NMR $\delta 137.5$ $(\mathrm{ArC}), 133.4\left(\mathrm{q}, J_{\mathrm{C}-\mathrm{F}}=35.0,2 \times \mathrm{CCF}_{3}\right), 130.8,128.7(\mathrm{ArCH}), 122.1\left(\mathrm{q}, J_{\mathrm{C}-\mathrm{F}}=274.8,2 \times \mathrm{CF}_{3}\right)$, $117.9(\mathrm{CN}), 61.5(\mathrm{CS}), 57.2(2 \times \mathrm{OCH}), 36.8\left(2 \times \mathrm{CH}_{2}\right)$.

$\left(1 R^{*}, 3 s, 5 S^{*}\right)$-Diethyl 3-[3,5-bis-(trifluoromethyl)phenylsulfonyl]-6-oxabicyclo[3.1.0] phosphonate (6e). White solid; $\mathrm{R}_{f}$ (hexane/EtOAc: 2/1) 0.13; mp 98-100 ${ }^{\circ} \mathrm{C}$; IR $v 3106$, 2982, 1601, 1368, 1288, 1126; ${ }^{1} \mathrm{H}$ NMR $\delta 8.45$ (s, 2H, ArH), 8.15 (s, 1H, ArH), 4.18-4.08 (m, 4H, 2 x $\left.\mathrm{OCH}_{2}\right), 3.75$ (s, $\left.2 \mathrm{H}, 2 \times \mathrm{OCH}\right), 2.84-2.58\left(\mathrm{~m}, 4 \mathrm{H}, 2 \times \mathrm{CH}_{2}\right), 1.26\left(\mathrm{t}, J=7.0,6 \mathrm{H}, 2 \times \mathrm{CH}_{3}\right) ;{ }^{13} \mathrm{C}$ 
NMR $\delta 139.9\left(\mathrm{~d}, J_{\mathrm{C}-\mathrm{P}}=2.2, \operatorname{ArC}\right), 132.2\left(\mathrm{q}, J_{\mathrm{C}-\mathrm{F}}=34.0,2 \times C \mathrm{CF}_{3}\right), 131.3,127.6(\mathrm{ArCH}), 122.4(\mathrm{q}$, $\left.J_{\mathrm{C}-\mathrm{F}}=273.3,2 \times \mathrm{CF}_{3}\right), 74.2\left(\mathrm{~d}, J_{\mathrm{C}-\mathrm{P}}=147.1, \mathrm{CP}\right), 64.1\left(\mathrm{~d}, J_{\mathrm{C}-\mathrm{P}}=6.6,2 \times \mathrm{OCH}_{2}\right), 59.2\left(\mathrm{~d}, J_{\mathrm{C}-\mathrm{P}}=4.4\right.$, $2 \times \mathrm{OCH}), 33.6\left(\mathrm{~d}, J_{\mathrm{C}-\mathrm{P}}=2.2,2 \times \mathrm{CCH}_{2}\right), 16.1\left(\mathrm{~d}, J_{\mathrm{C}-\mathrm{P}}=5.6,2 \times \mathrm{CH}_{3}\right) ; \mathrm{MS} m / z 496\left(\mathrm{M}^{+}, 0.04\right), 261$ (14), 219 (33), 218 (139), 213 (13), 189 (18), 163 (22), 161 (11), 149 (11), 83 (11), 82 (14), 81 (100), 65 (10), 53 (11); HRMS Calcd for $\mathrm{C}_{17} \mathrm{H}_{19} \mathrm{~F}_{6} \mathrm{O}_{6} \mathrm{SP}\left(\mathrm{M}^{+}\right)$496.0544, $\left(\mathrm{M}^{+}-\mathrm{F}\right)$ 477.0560, found: 477.0551.

$\left(1 R^{*}, 3 r, 5 S^{*}\right)$-Diethyl 3-[3,5-bis-(trifluoromethyl)phenylsulfonyl]-6-oxabicyclo[3.1.0] phosphonate (5e). White solid; $\mathrm{R}_{f}$ (hexane/EtOAc: 1/1) 0.12; mp 94-96 ${ }^{\circ} \mathrm{C}$; IR $v 3072$, 2916, 1701, 1573, 1411, 1288, 1155; ${ }^{1} \mathrm{H}$ NMR $\delta 8.39$ (s, 2H, ArH), 8.13 (s, 1H, ArH), 4.26-4.17 (m, 4H, 2 x $\mathrm{OCH}_{2}$ ), 3.55 (s, $2 \mathrm{H}, 2 \times \mathrm{OCH}$ ), 2.83-2.61 (m, 4H, $\left.2 \times \mathrm{CCH}_{2}\right), 1.32$ (t, $\left.J=6.9,6 \mathrm{H}, 2 \times \mathrm{CH}_{3}\right) ;{ }^{13} \mathrm{C}$ NMR $\delta 141.0\left(\mathrm{~d}, J_{\mathrm{C}-\mathrm{P}}=2.2, \operatorname{ArC}\right), 132.2\left(\mathrm{q}, J_{\mathrm{C}-\mathrm{F}}=34.0,2 \times C \mathrm{CF}_{3}\right), 131.1,127.4(\mathrm{ArCH}), 122.8(\mathrm{q}$, $\left.J_{\mathrm{C}-\mathrm{F}}=273.3,2 \times \mathrm{CF}_{3}\right), 73.7\left(\mathrm{~d}, J_{\mathrm{C}-\mathrm{P}}=136.1, \mathrm{CP}\right), 64.3\left(\mathrm{~d}, J_{\mathrm{C}-\mathrm{P}}=6.7,2 \times \mathrm{OCH}_{2}\right), 58.2\left(\mathrm{~d}, J_{\mathrm{C}-\mathrm{P}}=6.7\right.$, $2 \times \mathrm{OCH}), 34.0\left(\mathrm{~d}, J_{\mathrm{C}-\mathrm{P}}=1.5,2 \times \mathrm{CH}_{2}\right), 16.2\left(\mathrm{~d}, J_{\mathrm{C}-\mathrm{P}}=5.4,2 \times \mathrm{CH}_{3}\right) ; \mathrm{MS} m / z 496\left(\mathrm{M}^{+}, 0.69\right), 432$ (14), 261 (26), 219 (82), 218 (27), 213 (23), 194 (10), 191 (17), 190 (17), 189 (38), 163 (57), 162 (14), 161 (23), 145 (29), 135 (23), 133 (16), 109 (17), 83 (15), 82 (20), 81 (100), 65 (14), 53 (17); HRMS Calcd for $\mathrm{C}_{17} \mathrm{H}_{19} \mathrm{~F}_{6} \mathrm{O}_{6} \mathrm{SP}\left(\mathrm{M}^{+}\right)$496.0544, found: 496.0537.

General procedure for epoxide opening with azide. To a DMF (5 mL) solution of the corresponding epoxide $(0.5 \mathrm{mmol})$, an aqueous $20 \% \mathrm{v} / \mathrm{v}$ solution of $\mathrm{LiN}_{3}(1.2 \mathrm{~mL}, 5 \mathrm{mmol})$ or $\mathrm{NaN}_{3}$ (325 mg, $5 \mathrm{mmol}$ ) was added. The mixture was heated at $80{ }^{\circ} \mathrm{C}$ for $24-48 \mathrm{~h}$. The reaction was quenched with a saturated solution of $\mathrm{NH}_{4} \mathrm{Cl}(15 \mathrm{~mL})$, extracted with EtOAc $(2 \times 15 \mathrm{~mL})$, the organic phase was washed with $\mathrm{H}_{2} \mathrm{O}(2 \times 15 \mathrm{~mL})$ and dried $\left(\mathrm{MgSO}_{4}\right)$. Filtration and solvent evaporation afforded the corresponding crude azido alcohols, which were purified by flash chromatography (hexane/EtOAc) to afford pure compounds.

$\left(1 R^{*}, 2 R^{*}, 4 R^{*}\right)$-2-Azido-4-[3,5-bis-(trifluoromethyl)phenylsulfonyl]-4-phenylcyclopentanol

(7aa). Beige solid; $\mathrm{R}_{f}$ (hexane/EtOAc: 2/1) 0.44; mp 61-63 ${ }^{\circ} \mathrm{C}$; IR v 3467, 3082, 2926, 2109, 1616, 1598, 1459, 1351, 1279, 1147; ${ }^{1} \mathrm{H}$ NMR $\delta 8.0$ (s, 1H, ArH), 7.56 (s, 2H, ArH), 7.38-7.25 (m, 3H, ArH), 7.11 (d, $J=7.5,2 \mathrm{H}, \mathrm{ArH}), 4.65-4.59(\mathrm{~m}, 1 \mathrm{H}, \mathrm{CHOH}), 3.77-3.70\left(\mathrm{~m}, 1 \mathrm{H}, \mathrm{CHN}_{3}\right), 3.03$ (dd, $J$ $=14.5,7.1,1 \mathrm{H} \times \mathrm{CH}_{2} \mathrm{CHOH}$ ), 2.87 (d, $J=9.1,2 \mathrm{H}, \mathrm{CH}_{2} \mathrm{CHN}_{3}$ ), 2.35 (dd, $J=14.3,9.3,1 \mathrm{H} \mathrm{x}$ $\mathrm{CH}_{2} \mathrm{CHOH}$ ); ${ }^{13} \mathrm{C}$ NMR $\delta$ 137.1, 136.2 (ArC), 132.1 (q, $\left.J_{\mathrm{C}-\mathrm{F}}=35.0,2 \times \mathrm{CCF}_{3}\right), 130.1,129.5,128.7$, 128.4, 127.2 (ArCH), 122.1 (q, $\left.J_{\mathrm{C}-\mathrm{F}}=273.5,2 \times \mathrm{CF}_{3}\right), 75.5(\mathrm{CHOH}), 72.8(\mathrm{CS}), 65.7\left(\mathrm{CHN}_{3}\right), 39.6$ $\left(\mathrm{CH}_{2} \mathrm{CHOH}\right), 35.2\left(\mathrm{CH}_{2} \mathrm{CHN}_{3}\right)$; $\mathrm{MS} m / z 202$ (M+ $\left.\mathrm{BTFPSO}_{2}, 72\right), 159$ (79), 156 (21), 145 (22), 144 (579), 141 (15), 131 (24), 130 (25), 129 (54), 128 (259), 119 (39), 118 (22), 117 (34), 116 (17), 115 (56), 105 (11), 104 (30), 91 (100), 79 (13), 78 (16), 77 (45), 51 (12), 43 (12), 41 (16); HRMS Calcd for $\mathrm{C}_{19} \mathrm{H}_{15} \mathrm{~F}_{6} \mathrm{~N}_{3} \mathrm{O}_{3} \mathrm{~S}\left(\mathrm{M}^{+}\right)$479.0738, $\left(\mathrm{M}^{+}-\mathrm{H}_{2} \mathrm{O}-\mathrm{N}_{2}\right)$ 433.0577, found: 433.0571.

COSY summary: the following crossing peaks are observed: $\mathrm{H}_{\mathrm{A}}$ and $\mathrm{H}_{\mathrm{C}} ; \mathrm{H}_{\mathrm{B}}$ and $\mathrm{H}_{\mathrm{C}} ; \mathrm{H}_{\mathrm{C}}$ and $\mathrm{H}_{\mathrm{D}} ; \mathrm{H}_{\mathrm{D}}$ and $\mathrm{H}_{\mathrm{E}}, \mathrm{H}_{\mathrm{F}} ; \mathrm{H}_{\mathrm{E}}$ and $\mathrm{H}_{\mathrm{F}}$.

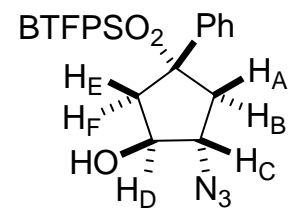

$\left(1 R^{*}, 2 R^{*}, 4 S^{*}\right)$-2-Azido-4-[3,5-bis-(trifluoromethyl)phenylsulfonyl]-4-phenylcyclopentanol

(8aa). Beige solid; $\mathrm{R}_{f}$ (hexane/EtOAc: 4/1) 0.25; mp 79-80 ${ }^{\circ} \mathrm{C}$; IR v3442, 3082, 2929, 2115, 1629, 1279, 1137; ${ }^{1} \mathrm{H}$ NMR $\delta 8.02$ (s, 1H, ArH), 7.55 (s, 2H, ArH), 739-7.26 (m, 3H, ArH), 7.07 (d, $J=$ 
7.5, 2H, ArH), 4.34-4.30 (m, 1H, $\mathrm{CHN}_{3}$ ), 4.21 (bs, $1 \mathrm{H}, \mathrm{CHOH}$ ), 3.22 (dd, $J=15.0,6.6,1 \mathrm{H} \mathrm{x}$ $\mathrm{CH}_{2} \mathrm{CHN}_{3}$ ), 2.87 (d, $J=6.2,2 \mathrm{H}, \mathrm{CH}_{2} \mathrm{CHOH}$ ), 2.44 (dd, $J=15.0,6.4,1 \mathrm{H} \mathrm{x} \mathrm{CH}_{2} \mathrm{CHN}_{3}$ ); ${ }^{13} \mathrm{C}$ NMR $\delta$ 136.9, 136.2 (ArC), 132.3 (q, $J_{\mathrm{C}-\mathrm{F}}=35.0,2$ x $\mathrm{CCF}_{3}$ ), 130.2, 129.6, 128.8, 128.6, $127.4(\mathrm{ArCH})$, $122.0\left(\mathrm{q}, J_{\mathrm{C}-\mathrm{F}}=273.5,2 \times \mathrm{CF}_{3}\right), 76.1(\mathrm{CHOH}), 75.9(\mathrm{CS}), 67.8\left(\mathrm{CHN}_{3}\right), 40.1\left(\mathrm{CH}_{2} \mathrm{CHOH}\right), 37.1$ $\left(\mathrm{CH}_{2} \mathrm{CHN}_{3}\right)$; $\mathrm{MS} m / z 213\left(\mathrm{M}^{+}-\mathrm{C}_{11} \mathrm{H}_{12} \mathrm{~N}_{3} \mathrm{O}_{3} \mathrm{~S}, 21\right), 203$ (12), 202 (100), 145 (23), 144 (60), 129 (44), 119 (43), 115 (40), 103 (48), 91 (80), 77 (34); HRMS Calcd for $\mathrm{C}_{19} \mathrm{H}_{15} \mathrm{~F}_{6} \mathrm{~N}_{3} \mathrm{O}_{3} \mathrm{~S}\left(\mathrm{M}^{+}\right.$) 479.0738, $\left(\mathrm{M}^{+}-\mathrm{H}_{2} \mathrm{O}-\mathrm{F}\right)$ 442.0649, found: 442.0692.

COSY summary: the following crossing peaks are observed: $\mathrm{H}_{\mathrm{A}}$ and $\mathrm{H}_{\mathrm{C}} ; \mathrm{H}_{\mathrm{B}}$ and $\mathrm{H}_{\mathrm{C}} ; \mathrm{H}_{\mathrm{D}}$ and $\mathrm{H}_{\mathrm{E}}, \mathrm{H}_{\mathrm{F}}$.

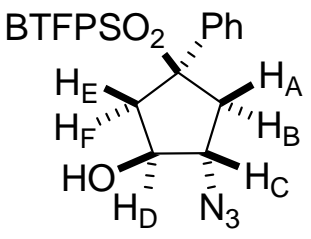

$\left(1 R^{*}, 3 R^{*}, 4 R^{*}\right)$-Isopropyl 3-azido-1-[3,5-bis-(trifluoromethyl)phenylsulfonyl]-4-hydroxycyclopentanecarboxylate (7ca). White solid; $\mathrm{R}_{f}$ (hexane/EtOAc: $2 / 1$ ) 0.41 ; mp $90-93{ }^{\circ} \mathrm{C}$; IR $v$ 3410, 3075, 2994, 2964, 2944, 2152, 1736, 1629, 1355, 1340, 1320, 1274, 1132; ${ }^{1} \mathrm{H}$ NMR $\delta 8.29$ (s, $2 \mathrm{H}, \mathrm{ArCH}$ ), 8.18 (s, $1 \mathrm{H}, \mathrm{ArCH}), 4.96$ (sept., $J=6.3,1 \mathrm{H}, \mathrm{OCH}), 4.31-4.27$ (m, $1 \mathrm{H}, \mathrm{CHOH}), 3.91-$

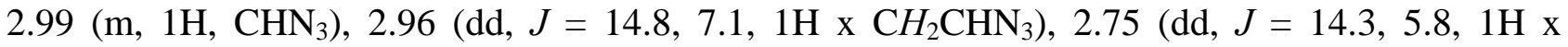
$\mathrm{CH}_{2} \mathrm{CHOH}$ ), 2.51-2.40 (m, $2 \mathrm{H}, 1 \times \mathrm{CH}_{2} \mathrm{CHN}_{3}, 1 \times \mathrm{CH}_{2} \mathrm{CHOH}$ ), 1.22 (d, $J=6.2,6 \mathrm{H}, 2 \times \mathrm{CH}_{3}$ ); ${ }^{13} \mathrm{C}$ NMR $\delta 167.1(\mathrm{CO}), 139.2(\mathrm{ArC}), 132.8\left(\mathrm{q}, J_{\mathrm{C}-\mathrm{F}}=35.0,2 \times \mathrm{CCF}_{3}\right), 130.3,127.8(\mathrm{ArCH}), 122.2(\mathrm{q}$, $\left.J_{\mathrm{C}-\mathrm{F}}=273.5,2 \mathrm{x} \mathrm{CF}_{3}\right), 76.4(\mathrm{CS}), 76.0(\mathrm{CHOH}), 71.9(\mathrm{OCH}), 66.4\left(\mathrm{CHN}_{3}\right), 38.2\left(\mathrm{CH}_{2} \mathrm{CHOH}\right), 34.2$ $\left(\mathrm{CH}_{2} \mathrm{CHN}_{3}\right)$, 21.2, $21.1\left(\mathrm{CH}_{3}\right)$; $\mathrm{MS} m / z 430\left(\mathrm{M}^{+}-\mathrm{H}_{2} \mathrm{O}-\mathrm{N}_{2}, 7\right)$, 261 (32), 213 (44), 194 (16), 163 (10), 144 (10), 143 (10), 142 (92), 127 (11), 125 (13), 124 (62), 115 (13), 114 (48), 98 (10), 97 (100), 96 (74), 69 (32), 68 (19), 53 (11), 43 (52), 41 (37); HRMS Calcd for $\mathrm{C}_{17} \mathrm{H}_{17} \mathrm{~F}_{6} \mathrm{~N}_{3} \mathrm{O}_{5} \mathrm{~S}\left(\mathrm{M}^{+}\right.$) 489.0793, $\left(\mathrm{M}^{+}-\mathrm{C}_{9} \mathrm{H}_{14} \mathrm{~N}_{3} \mathrm{O}_{3}\right)$ 276.9758, found: 276.9757.

COSY summary: the following crossing peaks are observed: $\mathrm{H}_{\mathrm{A}}$ and $\mathrm{H}_{\mathrm{C}} ; \mathrm{H}_{\mathrm{B}}$ and $\mathrm{H}_{\mathrm{C}}, \mathrm{H}_{\mathrm{C}}$ and $\mathrm{H}_{\mathrm{D}}, \mathrm{H}_{\mathrm{D}}$ and $\mathrm{H}_{\mathrm{E}}, \mathrm{H}_{\mathrm{F}}$.

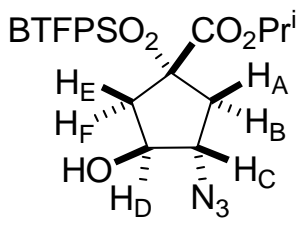

$\left(1 R^{*}, 2 R^{*}, 4 S^{*}\right)$-2-Azido-4-[3,5-bis-(trifluoromethyl)phenylsulfonyl]cyclopentanol (9). White solid; $\mathrm{R}_{f}$ (hexane/EtOAc: 2/1) 0.30; mp 96-98 ${ }^{\circ} \mathrm{C}$; IR v 3410, 3086, 2944, 2132, 2111, 1731, 1619, 1371, 1295, 1137; ${ }^{1} \mathrm{H}$ NMR $\delta 8.36$ (s, 2H, ArH), 8.17 (s, $\left.1 \mathrm{H}, \mathrm{ArH}\right), 4.38-4.26$ (m, $1 \mathrm{H}, \mathrm{CHOH}$ ), 3.36-3.45 (m 2H, CHS, $\mathrm{CHN}_{3}$ ), 2.47-2.37 (m, 2H, $\left.\mathrm{CH}_{2} \mathrm{CHOH}\right), 2.18-2.11,1.98-1.92(2 \mathrm{~m}, 2 \mathrm{H}$, $\left.\mathrm{CH}_{2} \mathrm{CHN}_{3}\right) ;{ }^{13} \mathrm{C}$ NMR $\delta 141.1(\mathrm{ArC}), 133.4\left(\mathrm{q}, J_{\mathrm{C}-\mathrm{F}}=35.0,2 \times \mathrm{CCF}_{3}\right), 128.8,127.6(\mathrm{ArCH}), 122.2$ (q, $\left.J_{\mathrm{C}-\mathrm{F}}=273.5,2 \times \mathrm{CF}_{3}\right), 75.9(\mathrm{CHOH}), 66.6\left(\mathrm{CHN}_{3}\right), 60.3(\mathrm{CHS}), 32.9\left(\mathrm{CH}_{2} \mathrm{CHOH}\right), 29.5$ $\left(\mathrm{CH}_{2} \mathrm{CHN}_{3}\right)$; MS m/z $277\left(\mathrm{M}^{+}-\mathrm{C}_{5} \mathrm{H}_{8} \mathrm{~N}_{3} \mathrm{O}\right.$, 5), 261 (36), 214 (18), 213 (66), 195 (13), 194 (28), 163 (26), 149 (20), 144 (21), 143 (13), 98 (100), 97 (18), 96 (12), 83 (45), 71 (77), 70 (33), 69 (23), 68 (21), 55 (13), 53 (13), 43 (75), 42 (13), 41 (41); HRMS Calcd for $\mathrm{C}_{13} \mathrm{H}_{11} \mathrm{~F}_{6} \mathrm{~N}_{3} \mathrm{O}_{3} \mathrm{~S}\left(\mathrm{M}^{+}\right)$403.0425, $\left(\mathrm{M}^{+}-\mathrm{C}_{5} \mathrm{H}_{8} \mathrm{~N}_{3} \mathrm{O}\right)$ 276.9758, found: 276.9761. 
$\left(1 R^{*}, 2 R^{*}, 4 R^{*}\right)$-2-Azido-4-[3,5-bis-(trifluoromethyl)phenylsulfonyl]cyclopentyl isopropyl carbonate (10). Colorless oil; $\mathrm{R}_{f}$ (pentane/EtOAc: 6/1) 0.53; IR $v$ 3085, 2989, 2934, 2112, 1744, 1618, 1285, 1149; ${ }^{1} \mathrm{H}$ NMR (mixture of rotamers) $\delta 8.35$ (s, 2H, ArH), 8.18 (s, 1H, ArH), 5.00-4.82 [m, 2H, OCH, OCH( $\left.\mathrm{CH}_{3}\right)_{2}$ ], 4.21-4.08 (m, 1H, CHN $)$, 3.81-3.7 (m, 1H, CHS), 2.59-2.41, 2.30$1.99\left(2 \mathrm{~m}, 2 \mathrm{H}, \mathrm{CH}_{2}\right), 1.30,1.26\left(2 \mathrm{~d}, J=4.9,3 \mathrm{H}, \mathrm{CH}_{3}\right) ;{ }^{13} \mathrm{C}$ NMR (mixture of rotamers) $\delta 153.4$, 153.1 (CO), 141.0, 140.7 (ArC), 133.4 (q, $J_{\mathrm{C}-\mathrm{F}}=33.1,2$ x $C C_{3}$ ), 128.9, 127.7 (ArCH), 122.2 (q, $J_{\mathrm{C}-}$ $\mathrm{F}=$ 273.3, $\left.2 \times \mathrm{CF}_{3}\right), 80.6,79.5(\mathrm{CHO}), 73.17,73.05\left[\mathrm{OCH}\left(\mathrm{CH}_{3}\right)_{2}\right], 64.7,64.4\left(\mathrm{CHN}_{3}\right), 61.1,60.5$ (CHS), 31.3, 30.59, 30.55, $30.3\left(\mathrm{CH}_{2}\right), 21.6,21.5\left(\mathrm{CH}_{3}\right)$; MS m/z $473\left(\mathrm{M}^{+}, 0.06\right), 279(17), 277$ (45), 262 (21), 261 (40), 214 (12), 213 (79), 194 (16), 163 (11), 98 (21), 97 (16), 96 (17), 81 (11), 80 (100), 71 (13), 69 (13), 68 (17), 53 (17), 43 (100), 41 (26); HRMS Calcd for $\mathrm{C}_{17} \mathrm{H}_{17} \mathrm{~F}_{6} \mathrm{~N}_{3} \mathrm{O}_{4} \mathrm{~S}$ $\left(\mathrm{M}^{+}\right)$473.0844, $\left(\mathrm{M}^{+}-\mathrm{Pr}^{\mathrm{i}}\right)$ 430.0296, found: 430.0282 .

General procedure for epoxide opening with benzylamine. Benzylamine $(1.1 \mathrm{~mL}, 10 \mathrm{mmol})$ was added to a DMF $(5 \mathrm{~mL})$ solution of epoxide $\mathbf{6 a}(0.5 \mathrm{mmol})$ and the reaction mixture was heated at $100{ }^{\circ} \mathrm{C}$ for $24 \mathrm{~h}$. The reaction was quenched with a saturated solution of $\mathrm{NH}_{4} \mathrm{Cl}(10 \mathrm{~mL})$, extracted with EtOAc ( $2 \times 10 \mathrm{~mL})$, and the organic phase dried $\left(\mathrm{MgSO}_{4}\right)$. Solvent evaporation afforded the corresponding crude alcohol amine, which was purified by flash chromatography to afford pure compound 7ab.

General procedure for epoxide opening with amines under solventless conditions. A mixture of the corresponding epoxide $\mathbf{6 a}$ or $\mathbf{6 d}(0.3 \mathrm{mmol})$ and benzyl or butyl amine $(1 \mathrm{~mL})$ was heated at 80 ${ }^{\circ} \mathrm{C}$ for $38 \mathrm{~h}$. The reaction was quenched with a saturated solution of $\mathrm{NH}_{4} \mathrm{Cl}(10 \mathrm{~mL})$, extracted with EtOAc ( $2 \times 10 \mathrm{~mL})$, and the organic phase was dried $\left(\mathrm{MgSO}_{4}\right)$. Solvent evaporation afforded the corresponding crude alcohol amine, which was purified by flash chromatography (hexane/EtOAc) to afford pure compounds $7 \mathbf{a b}, 7 \mathbf{a c}$ and $\mathbf{7 d b}$.

Experimental procedure for synthesis of compound 7ab under microwave irradiation. A 10 $\mathrm{mL}$ glass tube was charged with epoxide 6a $(0.3 \mathrm{mmol})$ and benzyl amine $(1 \mathrm{~mL})$, sealed with a septum and heated at $95{ }^{\circ} \mathrm{C}(200 \mathrm{~W})$ for $0.5 \mathrm{~h}$ with air stream cooling. The reaction mixture was cooled at room temperature and quenched with a saturated solution of $\mathrm{NH}_{4} \mathrm{Cl}(10 \mathrm{~mL})$. The mixture was extracted with EtOAc $(2 \times 10 \mathrm{~mL})$ and the organic phase was dried $\left(\mathrm{MgSO}_{4}\right)$. Filtration and solvent evaporation afforded the corresponding crude alcohol amine, which was purified by flash chromatography (hexane/EtOAc) to afford pure compound 7ab.

$\left(1 R^{*}, 2 R^{*}, 4 R^{*}\right)$-2-(Benzylamino)-4-[3,5-bis-(trifluoromethyl)phenylsulfonyl]-4-phenylcyclopentanol (7ab). White solid; $\mathrm{R}_{f}$ (hexane/EtOAc: 1/1) 0.12; mp 168-169 ${ }^{\circ} \mathrm{C}$; IR v 3300, 3074, 2927, 2873, 2814, 1626, 1444, 1365, 1287, 1140; ${ }^{1} \mathrm{H}$ NMR $\delta 7.98$ (s, 1H, ArH), 7.53 (s, 2H, ArH), 7.35-7.23 (m, 10H, ArH), 7.08 (d, $J=8.0,2 \mathrm{H}, \mathrm{ArH}), 4.49-4.43$ (m, 1H, CHOH), 3.94, 3.85 (2d, $J$ = 14.0, 2H, $\mathrm{CH}_{2} \mathrm{Ph}$ ), 3.05-2.94 (m, 2H, CHN, 1 x CH $\mathrm{CHOH}_{2} \mathrm{CHO}$ 2.80-2.66 (m, 2H, CH $\mathrm{CHN}_{2}, 2.29$ (dd, $\left.J=14.2,8.7,1 \mathrm{H} \times \mathrm{CH}_{2} \mathrm{CHOH}\right) ;{ }^{13} \mathrm{C}$ NMR $\delta$ 139.6, $137.5,136.9$ (ArC), 132.0 (q, $J_{\mathrm{C}-\mathrm{F}}=35.0,2$ x $\left.C_{C F}\right), 130.1,129.3,128.9,128.6,128.4,128.2,127.3,127.0(\mathrm{ArCH}), 122.1$ (q, $J_{\mathrm{C}-\mathrm{F}}=273.5,2 \mathrm{x}$ $\left.\mathrm{CF}_{3}\right), 76.1(\mathrm{CHOH}), 73.9(\mathrm{CS}), 64.4(\mathrm{CHN}), 52.4\left(\mathrm{CH}_{2} \mathrm{Ph}\right), 40.0\left(\mathrm{CH}_{2} \mathrm{CHOH}\right), 37.1\left(\mathrm{CH}_{2} \mathrm{CHN}\right)$; MS m/z 543 ( $\left.\mathrm{M}^{+}, 0.09\right), 266$ (44), 248 (18), 222 (19), 120 (15), 106 (44), 91 (100); HRMS Calcd for $\mathrm{C}_{26} \mathrm{H}_{23} \mathrm{~F}_{6} \mathrm{NO}_{3} \mathrm{~S}\left(\mathrm{M}^{+}\right)$543.1303, $\left(\mathrm{M}^{+}-\mathrm{BTFPSO}_{2}\right)$ 266.1545, found: 266.1512.

$\left(1 R^{*}, 3 R^{*}, 4 R^{*}\right)$-3-(Benzylamino)-1-[3,5-bis-(trifluoromethyl)phenylsulfonyl]-4-hydroxy-1cyanocyclopentane (7db). White solid. $\mathrm{R}_{f}$ (hexane/EtOAc: 1/1) 0.43; mp 162-163 ${ }^{\circ} \mathrm{C}$; IR $v 3446$, 3258, 3086, 2918, 2842, 1624, 1604, 1467, 1345, 1284, 1137; ${ }^{1} \mathrm{H}$ NMR $\delta 8.45$ (s, 2H, ArH), 8.26 (s, 1H, ArH), 7.37-7.27 (m, 5H, ArH), 4.27-4.22 (m, 1H, CHOH), 3.87, 381 (2d, $J=13.3,2 \mathrm{H}$, 
$\mathrm{CH}_{2} \mathrm{Ph}$ ), 3.31 (m, 1H, CHN), 2.82 (dd, $J=14.3,6.0,1 \mathrm{H}$ x CH $\mathrm{CH}_{2} \mathrm{CHOH}$ ), 2.61, 2.35 (2dd, $J=14.3$, 6.6, 2H, $\mathrm{CH}_{2} \mathrm{CHN}$ ), 2.19 (dd, $J=14.2,4.5,1 \mathrm{H}$ x CH $\mathrm{CHOH}_{2} \mathrm{CH}{ }^{13} \mathrm{C}$ NMR $\delta$ 139.3, 137.3 (ArC), 134.0 (q, $\left.J_{\mathrm{C}-\mathrm{F}}=34.0,2 \times \mathrm{CCF}_{3}\right), 130.6,128.7,128.0,127.5(\mathrm{ArCH}), 122.1$ (q, $\left.J_{\mathrm{C}-\mathrm{F}}=273.3,2 \times \mathrm{CF}_{3}\right), 118.1$ $(\mathrm{CN}), 76.1(\mathrm{CHOH}), 64.8(\mathrm{CS}), 63.7(\mathrm{CHN}), 52.3\left(\mathrm{CH}_{2} \mathrm{Ph}\right), 39.6\left(\mathrm{CH}_{2} \mathrm{CHOH}\right), 37.7\left(\mathrm{CH}_{2} \mathrm{CHN}\right)$; MS m/z $492\left(\mathrm{M}^{+}, 0.01\right), 215$ (26), 171 (24), 91 (10); HRMS Calcd for $\mathrm{C}_{21} \mathrm{H}_{18} \mathrm{~F}_{6} \mathrm{~N}_{2} \mathrm{O}_{3} \mathrm{~S}\left(\mathrm{M}^{+}\right)$ 492.0942, found: 492.0948.

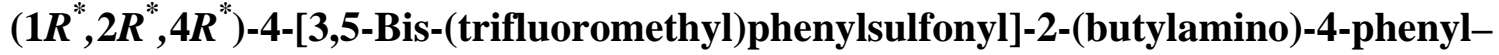

cyclopentanol (7ac). Beige solid; $\mathrm{R}_{f}$ (hexane/EtOAc: 1/4) 0.23; mp 173-174 ${ }^{\circ} \mathrm{C}$; IR $v$ 3291, 2959, 2919, 2847, 1451, 1357, 1308, 1276, 1133; ${ }^{1} \mathrm{H}$ NMR $\delta 7.98$ (s, 1H, ArH), 7.55 (s, 2H, ArH), 7.35 (m, 3H, ArH), 7.13 (d, $J=7.6,2 \mathrm{H}, \mathrm{ArH}), 4.48-4.40$ (m, 1H, CHOH), 2.99-2.28 (m, 7H, CHN, $\mathrm{CH}_{2} \mathrm{CHOH}, \mathrm{CH}_{2} \mathrm{CHN}, \mathrm{NCH}_{2} \mathrm{CH}_{2}$ ), $1.58-1.33\left(\mathrm{~m}, 4 \mathrm{H}, 2 \times \mathrm{CH}_{2}\right), 0.94\left(\mathrm{t}, J=7.3,3 \mathrm{H}, \mathrm{CH}_{3}\right) ;{ }^{13} \mathrm{C}$ NMR $\delta$ 137.5, 137.1 (ArC), 132.0 (q, $\left.J_{\mathrm{C}-\mathrm{F}}=34.0,2 \times \mathrm{CCF}_{3}\right), 130.2,129.2,129.0,128.4,126.9$ $(\mathrm{ArCH}), 122.2$ (q, $\left.J_{\mathrm{C}-\mathrm{F}}=273.3,2 \times \mathrm{CF}_{3}\right), 75.7(\mathrm{CHOH}), 74.0(\mathrm{CS}), 64.9(\mathrm{CHN}), 48.0,40.2,37.2$, 32.4, $20.4\left(\mathrm{CH}_{2}\right), 13.9\left(\mathrm{CH}_{3}\right) ; \mathrm{MS} \mathrm{m} / z 509$ (M ${ }^{+}$, 0.81), 466 (19), 233 (17), 232 (100), 214 (36), 213 (12), 202 (10), 189 (10), 188 (63), 177 (14), 170 (41), 159 (14), 158 (11), 149 (55), 144 (21), 141 (14), 129 (13), 115 (19), 111 (12), 105 (13), 97 (16), 95 (11), 91 (22), 86 (30), 85 (15), 84 (12), 83 (15), 77 (11), 72 (17), 71 (15), 69 (15), 57 (38), 55 (19), 44 (14), 43 (19), 41 (15); HRMS Calcd for $\mathrm{C}_{23} \mathrm{H}_{25} \mathrm{~F}_{6} \mathrm{NO}_{3} \mathrm{~S}\left(\mathrm{M}^{+}\right)$509.1459, found: 509.1484.

General procedure for epoxide ring opening with bromodimethylsulfonium bromide. To a solution of the corresponding epoxide $(0.1 \mathrm{mmol})$ in $\mathrm{CH}_{3} \mathrm{CN}(0.8 \mathrm{~mL})$ stirred at $\mathrm{RT}$ bromodimethylsulfonium $^{16}$ (71 mg, $0.5 \mathrm{mmol}$ ) was added portionwise. After stirring at RT for $16 \mathrm{~h}$ the mixture was quenched with $\mathrm{H}_{2} \mathrm{O}(2 \mathrm{~mL})$, extracted with EtOAc $(2 \times 2 \mathrm{~mL})$, and the organic phase dried $\left(\mathrm{MgSO}_{4}\right)$. Solvent evaporation afforded the corresponding crude bromo alcohols, which were purified by flash chromatography (hexane/EtOAc) to afford pure compounds.

$\left(1 R^{*}, 2 R^{*}, 4 R^{*}\right)$-4-[3,5-bis-(trifluoromethyl)phenylsulfonyl]-2-bromo-4-phenylcyclopentanol

(7ad). White solid; $\mathrm{R}_{f}$ (hexane/EtOAc: 4/1) 0.28; mp 125-127 ${ }^{\circ} \mathrm{C}$; IR $v$ 3540, 3083, 1620, 1597, 1352, 1282, 1126; ${ }^{1} \mathrm{H}$ NMR $\delta 8.00$ (s, 1H, ArH), 7.56 (s, 2H, ArH), 7.40-7.25 (m, 3H, ArH), 7.12 (d, $J=7.7,2 \mathrm{H}, \mathrm{ArH}), 4.80-4.70(\mathrm{~m}, 1 \mathrm{H}, \mathrm{CHOH}), 3.93-3.84(\mathrm{~m}, 1 \mathrm{H}, \mathrm{CHBr}), 3.26$ (dd, $J=14.6$, 10.8, $1 \mathrm{H} \times \mathrm{CH}_{2} \mathrm{CHBr}$ ), 3.11 (dd, $J=14.7,8.1,1 \mathrm{H} \times \mathrm{CH}_{2} \mathrm{CHBr}$ ), 3.03 (dd, $J=14.4,7.4,1 \mathrm{H} \times$ $\left.\mathrm{CH}_{2} \mathrm{CHOH}\right), 2.50(\mathrm{~d}, J=3.9,1 \mathrm{H}, \mathrm{OH}), 2.33\left(\mathrm{dd}, J=14.5,9.2,1 \mathrm{H} \times \mathrm{CH}_{2} \mathrm{CHOH}\right) ;{ }^{13} \mathrm{C} \mathrm{NMR} \delta$ 137.1, 135.8 (ArC), 132.2 (q, $\left.J_{\mathrm{C}-\mathrm{F}}=34.6,2 \times \mathrm{CCF}_{3}\right), 130.2,129.6,128.8,128.6,127.2(\mathrm{ArCH})$, $122.1\left(\mathrm{q}, J_{\mathrm{C}-\mathrm{F}}=273.9,2 \times \mathrm{CF}_{3}\right), 78.2(\mathrm{CHOH}), 73.7(\mathrm{CS}), 50.8(\mathrm{CHBr}), 39.8\left(\mathrm{CH}_{2} \mathrm{CHBr}\right), 39.5$ $\left(\mathrm{CH}_{2} \mathrm{CHOH}\right) ; \mathrm{MS} \mathrm{m} / \mathrm{z} 241\left(\mathrm{M}^{+}+2-\mathrm{BTFPSO}_{2}, 100\right), 239\left(\mathrm{M}^{+}-\mathrm{BTFPSO}_{2}, 100\right), 223$ (100), 221 (100), 213 (32), 163 (13), 160 (24), 159 (100), 144 (24), 143 (32), 142 (100), 141 (64), 131 (100), 129 (33), 115 (81), 105 (20), 103 (34), 91 (64), 77 (26); HRMS Calcd for $\mathrm{C}_{19} \mathrm{H}_{15} \mathrm{BrF}_{6} \mathrm{O}_{3} \mathrm{~S}\left(\mathrm{M}^{+}\right)$ 515.9829, $\left(\mathrm{M}^{+}-\mathrm{BTFPSO}_{2}\right) 239.0072$ found: 239.0072.

$\left(1 R^{*}, 3 R^{*}, 4 S^{*}\right)$-4-[3,5-bis-(trifluoromethyl)phenylsulfonyl]-2-bromo-4-phenylcyclopentanol

(8ad). White solid; $\mathrm{R}_{f}$ (hexane/EtOAc: 2/1) 0.51; mp 153-155 ${ }^{\circ} \mathrm{C}$; IR $v$ 3537, 3050, 1629, 1609, 1361, 1279, 1188, 1142; ${ }^{1} \mathrm{H}$ NMR $\delta 8.03$ (s, 1H, ArH), 7.55 (s, 2H, ArH), 7.40-7.26 (m, 3H, ArH), $7.10(\mathrm{~d}, J=7.8,2 \mathrm{H}, \mathrm{ArH}), 4.54-4.42(\mathrm{~m}, 2 \mathrm{H}, \mathrm{CHOH}, \mathrm{CHBr}), 3.56(\mathrm{dd}, J=15.5,6.5,1 \mathrm{H} \times$ $\mathrm{CH}_{2} \mathrm{CHBr}$ ), 3.51 (d, J = 7.8, 1H, OH), 3.08 (dd, $J=15.5,6.8,1 \mathrm{H} \mathrm{x} \mathrm{CH} \mathrm{CHBr}_{2}$ ) 2.92-2.83 (m, 2H, 1 $\left.\mathrm{x} \mathrm{CH} \mathrm{CHBr}_{2} 1 \mathrm{x} \mathrm{CH} \mathrm{CH}_{2} \mathrm{CHOH}\right) ;{ }^{13} \mathrm{C} \mathrm{NMR}\left(100 \mathrm{MHz}, \mathrm{C}_{6} \mathrm{D}_{6}\right) \delta 137.7,137.0$ (ArC), $132.0\left(\mathrm{q}, J_{\mathrm{C}-\mathrm{F}}=\right.$ 34.0, 2 x $\mathrm{CCF}_{3}$ ), 130.3, 129.19, 129.12, 128.3, 126.9 (ArCH), 122.5 (q, $\left.J_{\mathrm{C}-\mathrm{F}}=273.3,2 \times \mathrm{CF}_{3}\right), 78.9$ $(\mathrm{CHOH}), 76.3$ (CS), $53.3(\mathrm{CHBr}), 41.5\left(\mathrm{CH}_{2} \mathrm{CHBr}\right), 39.7\left(\mathrm{CH}_{2} \mathrm{CHOH}\right) ; \mathrm{MS} \mathrm{m} / z 241\left(\mathrm{M}^{+}+2-\right.$ 
$\left.\mathrm{BTFPSO}_{2}, 62\right), 239$ ( $\left.\mathrm{M}^{+}-\mathrm{BTFPSO}_{2}, 62\right), 223$ (46), 221 (45), 213 (15), 159 (99), 144 (17), 142 (100), 141 (37), 131 (62), 129 (27), 115 (55), 105 (14), 103 (19), 91 (45), 77 (19), 43 (13); HRMS Calcd for $\mathrm{C}_{19} \mathrm{H}_{15} \mathrm{BrF}_{6} \mathrm{O}_{3} \mathrm{~S}\left(\mathrm{M}^{+}\right)$515.9829, found: 515.98.38.

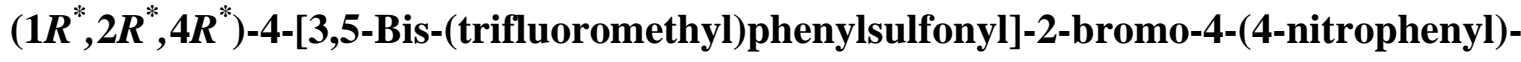
cyclopentanol (7bd). White solid; $\mathrm{R}_{f}$ (hexane/EtOAc: 7/3) 0.47; mp 249-250 ${ }^{\circ} \mathrm{C}$; IR $v$ 3507, 3081, 2969, 2918, 2842, 1731, 1609, 1513, 1361, 1274, 1142; ${ }^{1} \mathrm{H}$ NMR $\left(\mathrm{CD}_{3} \mathrm{COCD}_{3}\right) \delta 8.41$ (s, $\left.1 \mathrm{H}, \mathrm{ArH}\right)$, 8.15 (d, $J=8.9,2 \mathrm{H}, \mathrm{ArH}), 7.76$ (s, 2H, ArH), 7.83 (d, $J=8.8,2 \mathrm{H}, \mathrm{ArH}), 4.91$ (d, $J=5.4,1 \mathrm{H}, \mathrm{OH}$ ) 4.70-4.65 (m, $1 \mathrm{H}, \mathrm{CHOH}$ ), 4.13-4.07 (m, $1 \mathrm{H}, \mathrm{CHBr}$ ), 3.38 (dd, $J=15.1,8.1,1 \mathrm{H} \times \mathrm{CH}_{2} \mathrm{CHBr}$ ), 3.25-3.17 (m, 2H, $1 \times \mathrm{CH}_{2} \mathrm{CHBr}, 1 \times \mathrm{CH}_{2} \mathrm{CHOH}$ ), 2.45 (dd, $J=14.4,8.4,1 \mathrm{H} \times \mathrm{CH}_{2} \mathrm{CHOH}$ ); ${ }^{13} \mathrm{C}$ NMR (100MHz, $\left.\mathrm{CD}_{3} \mathrm{COCD}_{3}\right) \delta 148.9,144.7,138.1(\mathrm{ArC}), 132.9$ (q, $\left.J_{\mathrm{C}-\mathrm{F}}=35.0,2 \times \mathrm{CCF}_{3}\right), 131.8$, 131.2, 129.1, $123.8(\mathrm{ArCH}), 123.4\left(\mathrm{q}, J_{\mathrm{C}-\mathrm{F}}=272.1,2 \times \mathrm{CF}_{3}\right), 79.0(\mathrm{CHOH}), 75.4(\mathrm{CS}), 52.2$ (CHBr), $41.1\left(\mathrm{CH}_{2} \mathrm{CHBr}\right), 40.9\left(\mathrm{CH}_{2} \mathrm{CHOH}\right)$; $\mathrm{MS} \mathrm{m} / z 563\left(\mathrm{M}^{+}+2,0.23\right), 561\left(\mathrm{M}^{+}, 0.22\right), 286$ (32), 284 (36), 268 (55), 266 (54), 214 (15), 213 (25), 205 (26), 204 (100), 195 (14), 188 (35), 187 (94), 176 (20), 163 (18), 158 (54), 150 (12), 145 (14), 144 (15), 141 (25), 139 (14), 131 (19), 130 (53), 129 (51), 128 (57), 127 (22), 116 (479, 115 (82), 103 (16), 102 (21), 91 (16), 89 (14), 77 (15), 63 (13), 58 (17), 43 (13); HRMS Calcd for $\mathrm{C}_{19} \mathrm{H}_{14} \mathrm{BrF}_{6} \mathrm{NO}_{5} \mathrm{~S}\left(\mathrm{M}^{+}\right)$560.9680, found: 560.9674.

$\left(1 R^{*}, 3 R^{*}, 4 R^{*}\right)$-Isopropyl 1-[3,5-bis-(trifluoromethyl)phenylsulfonyl]-3-bromo-4-hydroxycyclopentanecarboxylate (7cd). Yellow oil; $\mathrm{R}_{f}$ (hexane/EtOAc: 4/1) 0.28; IR $v$ 3478, 3086, 2983, 2921, 1724, 1631, 1358, 1275, 1152; ${ }^{1} \mathrm{H}$ NMR $\delta 8.30$ (s, 2H, ArH), 8.18 (s, 1H, ArH), 4.95 (sept., $J$ $=6.2,1 \mathrm{H}, \mathrm{OCH}), 4.53-4.49(\mathrm{~m}, 1 \mathrm{H}, \mathrm{CHOH}), 4.04-3.99(\mathrm{~m}, 1 \mathrm{H}, \mathrm{CHBr}), 3.25$ (dd, $J=15.1,7.5,1 \mathrm{H}$ x $\mathrm{CH}_{2} \mathrm{CHBr}$ ), 2.94 (dd, $J=14.3,6.2,1 \mathrm{H} \times \mathrm{CH}_{2} \mathrm{CHOH}$ ), 2.86 (dd, $J=15.0,7.7,1 \mathrm{H} \times \mathrm{CH}_{2} \mathrm{CHBr}$ ), 2.45 (dd, $J=14.4,5.0,1 \mathrm{H} \times \mathrm{CH}_{2} \mathrm{CHOH}$ ), $2.25(\mathrm{~s}, 1 \mathrm{H}, \mathrm{OH}), 1.21$ (d, $\left.J=6.7,6 \mathrm{H}, 2 \times \mathrm{CH}_{3}\right) ;{ }^{13} \mathrm{C}$ NMR $\delta 166.9$ (CO), 139.2 (ArC), 132.8 (q, $\left.J_{\mathrm{C}-\mathrm{F}}=35.0,2 \times C \mathrm{CF}_{3}\right), 130.4,127.9(\mathrm{ArCH}), 122.2$ (q, $\left.J_{\mathrm{C}-\mathrm{F}}=273.5,2 \mathrm{x} \mathrm{CF}_{3}\right), 78.9(\mathrm{CHOH}), 76.8(\mathrm{CS}), 71.9(\mathrm{OCH}), 50.5(\mathrm{CHBr}), 38.8\left(\mathrm{CH}_{2} \mathrm{CHBr}\right), 37.6$ $\left(\mathrm{CH}_{2} \mathrm{CHOH}\right)$, 21.2, $21.1\left(\mathrm{CH}_{3}\right)$; MS m/z 467 (M $\left.{ }^{+}-\mathrm{OPr}^{\mathrm{i}}, 4.5\right), 422$ (11), 420 (14), 391 (10), 378 (53), 350 (13), 349 (66), 331 (39), 279 (13), 261 (50), 213 (42), 209 (27), 207 (30), 194 (18), 191 (74); 189 (77), 169 (23), 168 (27), 163 (23), 161 (13), 157 (12), 147 (15), 145 (19), 144 (12), 127 (53), 126 (47), 125 (23); 115 (12); 111 (28), 110 (12), 109 (33), 99 (16), 97 (18), 83 (35), 82 (23), 81 (39), 65 (14), 55 (19), 53 (34), 43 (100), 41 (23); HRMS Calcd for $\mathrm{C}_{17} \mathrm{H}_{17} \mathrm{BrF}_{6} \mathrm{O}_{5} \mathrm{~S}$ ( $\mathrm{M}^{+}$) 525.9884, ( $\left.\mathrm{M}^{+}-\mathrm{OH}\right) 508.9857$ found: 508.9852 .

$\left(1 R^{*}, 3 R^{*}, 4 R^{*}\right)-1$-[3,5-Bis-(trifluoromethyl)phenylsulfonyl]-3-bromo-4-hydroxy-1-cyano-

cyclopentane (7dd). White solid; $\mathrm{R}_{f}$ (hexane/EtOAc: 2/1) 0.46; mp 120-122 ${ }^{\circ} \mathrm{C}$; IR $v 3481$, 3082, 2934, 2863, 2257, 1623, 1607, 1279, 1148; ${ }^{1} \mathrm{H}$ NMR $\delta 8.48$ (s, 2H, ArH), 8.28 (s, $\left.1 \mathrm{H}, \mathrm{ArH}\right), 4.58-$ 4.56 (m, $1 \mathrm{H}, \mathrm{CHOH}), 4.22-4.16$ (m, 1H, CHBr), 3.10-2.90 (m, 3H, $\mathrm{CH}_{2} \mathrm{CHBr}, 1 \times \mathrm{CH}_{2} \mathrm{CHOH}$ ), 2.60 (s, $1 \mathrm{H}, \mathrm{OH}$ ), 2.29 (dd, $J=14.3,4.7,1 \mathrm{H}, 1 \times \mathrm{CH}_{2} \mathrm{CHOH}$ ); ${ }^{13} \mathrm{C}$ NMR $\delta 137.0$ (ArC), 133.7 (q, $\left.J_{\mathrm{C}-\mathrm{F}}=35.12 \times \mathrm{CCF}_{3}\right), 130.7,129.1(\mathrm{ArCH}), 122.0\left(\mathrm{q}, J_{\mathrm{C}-\mathrm{F}}=274.2,2 \times \mathrm{CF}_{3}\right), 117.5(\mathrm{CN}), 78.8(\mathrm{CS})$, $64.1(\mathrm{CHOH}), 49.0(\mathrm{CHBr}), 40.6\left(\mathrm{CH}_{2} \mathrm{CHBr}\right), 39.1\left(\mathrm{CH}_{2} \mathrm{CHOH}\right) ; \mathrm{MS} \mathrm{m} / z 467\left(\mathrm{M}^{+}+2,1.3\right), 465$ ( $\left.\mathrm{M}^{+}, 1.4\right), 448$ (11), 446 (10), 310 (26), 279 (22), 277 (29), 261 (23), 214 (10), 213 (100), 195 (11), 194 (34); 190 (76); 188 (78), 172 (40); 170 (39); 163 (28), 149 (14), 144 (25), 143 (13), 109 (20); 108 (48), 107 (38), 106 (11), 92 (18), 91 (29), 81 (21), 80 (66), 53 (48), 52 (10), 43 (15); HRMS Calcd for $\mathrm{C}_{14} \mathrm{H}_{10} \mathrm{BrNF}_{6} \mathrm{O}_{3} \mathrm{~S}\left(\mathrm{M}^{+}\right)$464.9469, found: 464.9467.

$\left(1 R^{*}, 3 R^{*}, 4 R^{*}\right)$-Diethyl 1-[3,5-bis-(trifluoromethyl)phenylsulfonyl]-3-bromo-4-hydroxycyclopentylphosphonate (7ed). Yellow oil; $\mathrm{R}_{f}$ (hexane/EtOAc: 1/1) 0.48; IR v 3396, 3107, 2988, 2937, 1626, 1446, 1358, 1270, 1146, 1028; ${ }^{1} \mathrm{H}$ NMR $\delta 8.44$ (s, 2H, ArH), 8.18 (s, 1H, ArH), 4.52- 
$4.43(\mathrm{~m}, 1 \mathrm{H}, \mathrm{CHOH}), 4.24-3.97$ (m, 5H, CHBr, 2 x $\mathrm{OCH}_{2}$ ), 3.35 (d, $\left.J=6.2,1 \mathrm{H}, \mathrm{OH}\right), 2.99-2.74$

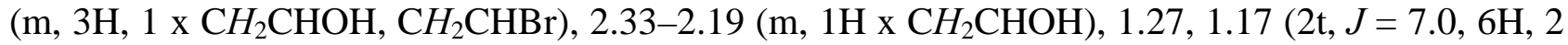
x $\left.\mathrm{CH}_{3}\right) ;{ }^{13} \mathrm{C}$ NMR $\delta 139.9\left(\mathrm{~d}, J_{\mathrm{C}-\mathrm{P}}=2.2, \operatorname{ArC}\right), 132.5\left(\mathrm{q}, J_{\mathrm{C}-\mathrm{F}}=33.7,2 \times \mathrm{CCF}_{3}\right), 131.1,127.7$ $(\mathrm{ArCH}), 122.3\left(\mathrm{q}, J_{\mathrm{C}-\mathrm{F}}=273.5,2 \times \mathrm{CF}_{3}\right), 78.5\left(\mathrm{~d}, J_{\mathrm{C}-\mathrm{P}}=5.4, \mathrm{CHOH}\right), 69.2\left(\mathrm{~d}, J_{\mathrm{C}-\mathrm{P}}=144.2, \mathrm{CS}\right)$, 64.8, $64.1\left(2 \mathrm{~d}, J_{\mathrm{C}-\mathrm{P}}=6.7,2 \times \mathrm{OCH}_{2}\right), 50.9\left(\mathrm{~d}, J_{\mathrm{C}-\mathrm{P}}=6.7, \mathrm{CHBr}\right), 37.4\left(\mathrm{CH}_{2} \mathrm{CHBr}\right), 35.9$ $\left(\mathrm{CH}_{2} \mathrm{CHOH}\right), 16.1,15.9$ (2d, $\left.J_{\mathrm{C}-\mathrm{P}}=6.7,2 \times \mathrm{CH}_{3}\right) ; \mathrm{MS} m / z 577$ (M $\left.{ }^{+}, 0.24\right), 442$ (13), 441 (83), 433 (25), 413 (13), 385 (19), 365 (38), 301(96), 299 (100), 283 (33), 281 (34), 273 (10), 271 (11), 261 (29), 255 (12), 253 (11), 243 (10), 227 (35), 225 (35), 219 (78), 218 (11), 213 (31), 207 (64), 194 (14), 191 (24), 187 (11), 163 (64), 161 (19), 155 (38), 147 (16), 146 (25), 145 (36), 135 (23), 133 (13), 127 (18), 109 (24), 99 (19), 83 (19), 82 (17), 81 (39), 65 (23), 53 (18); HRMS Calcd for $\mathrm{C}_{17} \mathrm{H}_{20} \mathrm{BrNF}_{6} \mathrm{O}_{6} \mathrm{PS}\left(\mathrm{M}^{+}\right)$575.9806, found: 575.9821.

General procedure for preparation of cyclopentenones 11-14. Method A (Swern oxidation). A mixture of $\mathrm{CH}_{2} \mathrm{Cl}_{2}(725 \mu \mathrm{L})$ and DMSO $(246 \mu \mathrm{L}, 3.2 \mathrm{mmol})$ was added within $5 \mathrm{~min}$ to a stirred solution of oxalyl chloride ( $145 \mu \mathrm{L}, 1.6 \mathrm{mmol})$ in $\mathrm{CH}_{2} \mathrm{Cl}_{2}(3.6 \mathrm{~mL})$ at $-60{ }^{\circ} \mathrm{C}$. The reaction mixture was stirred for $15 \mathrm{~min}$ and then the alcohol 7 (1.45 mmol) was added within $5 \mathrm{~min}$; stirring was continued for an additional $15 \mathrm{~min}$. TEA $(1 \mathrm{~mL}, 7.2 \mathrm{mmol})$ was then added, and the reaction mixture was stirred overnight and allowed to warm to room temperature. Water $(10 \mathrm{~mL})$ was then added and the aqueous layer was extracted with additional $\mathrm{CH}_{2} \mathrm{Cl}_{2}(2 \times 10 \mathrm{~mL})$. The organic layers were combined, washed with saturated $\mathrm{NaCl}$ solution $(20 \mathrm{~mL})$, and dried $\left(\mathrm{MgSO}_{4}\right)$. Solvent evaporation afforded the corresponding crude cyclopentenones, which were purified by flash chromatography (pentane/Et ${ }_{2} \mathrm{O}$ ).

Method B (TPAP/NMO). TPAP (27 $\mathrm{mg}, 0.075 \mathrm{mmol}$ ) was added to a stirred mixture of alcohol 7ad (273 mg, $0.5 \mathrm{mmol})$ and $\mathrm{NMO}(180 \mathrm{mg}, 1.5 \mathrm{mmol})$ in $\mathrm{CH}_{2} \mathrm{Cl}_{2}(2 \mathrm{~mL})$ at RT. After stirred for $24 \mathrm{~h}$ at the same temperature, the reaction mixture was filtered through a pad of Celite, which was washed with $\mathrm{Et}_{2} \mathrm{O}$. Solvent evaporation afforded the corresponding crude cyclopentenone, which was purified by flash chromatography (pentane/Et ${ }_{2} \mathrm{O}$ ).

Method C (IBX). To a solution of corresponding alcohol $(0.5 \mathrm{mmol})$ in THF (3 mL) and DMSO (3 $\mathrm{mL}$, for compound 7ad), or DMSO (6 mL, for $\mathbf{7 b d}$ and 7ca), was added IBX ${ }^{20}$ (418 mg, $1.5 \mathrm{mmol}$ ), and the mixture was stirred at RT for $48 \mathrm{~h}$. The mixture was diluted with $\mathrm{H}_{2} \mathrm{O}(4 \mathrm{~mL})$, and stirred at $0{ }^{\circ} \mathrm{C}$ for $10 \mathrm{~min}$. The resulting suspension was filtered through a Celite pad, which was washed with EtOAc. The filtrated was diluted with brine $(5 \mathrm{~mL})$, followed by extraction with $\mathrm{Et}_{2} \mathrm{O}(3 \times 15 \mathrm{ml})$ and dried $\left(\mathrm{MgSO}_{4}\right)$. Solvent evaporation afforded the corresponding crude cyclopentenones, which were purified by flash chromatography (pentane/ $\mathrm{Et}_{2} \mathrm{O}$ ).

5-Bromo-3-phenyl-2-cyclopentenone (11). White solid; $\mathrm{R}_{f}$ (pentane/Et $\mathrm{t}_{2} \mathrm{O}$ : 6/1) 0.23; mp 58-60 ${ }^{\circ} \mathrm{C}$; IR $v 2919,2850,1691,1598,1441 ;{ }^{1} \mathrm{H}$ NMR $\delta 7.65$ (d, $\left.J=7.0,2 \mathrm{H}, \operatorname{ArH}\right), 7.52-7.46$ (m, 3H, ArH), 6.65 (bs, $1 \mathrm{H}, \mathrm{CH}=\mathrm{C}$ ), 4.56 (dd, $J=6.8,2.2,1 \mathrm{H}, \mathrm{CHBr}$ ), 3.77-3.71 (m, $1 \mathrm{H} \mathrm{x} \mathrm{CH}$ ), 3.36 (dd, $J=$ 18.8, 1.6, $1 \mathrm{H} \times \mathrm{CH}_{2}$ ); ${ }^{13} \mathrm{C}$ NMR $\delta 201.9(\mathrm{CO}), 171.0,169.7$ (ArC, $\mathrm{CH}=\mathrm{C}$ ), 132.1, 129.0, 127.0, 124.2 ( $\mathrm{ArCH}, \mathrm{CH}=\mathrm{C}), 42.1(\mathrm{CHBr}), 40.2\left(\mathrm{CH}_{2}\right) ; \mathrm{MS} m / z 238\left(\mathrm{M}^{+}+2,16\right), 236\left(\mathrm{M}^{+}, 17\right), 158(14)$, 157 (100), 130 (38), 128 (62), 127 (24), 102 (20), 64 (11), 51 (12); HRMS Calcd for $\mathrm{C}_{11} \mathrm{H}_{9} \mathrm{BrO}$ $\left(\mathrm{M}^{+}\right)$235.9837, found: 235.9835

5-Bromo-3-(4-nitrophenyl)-2-cyclopentenone (13). White solid; $\mathrm{R}_{f}$ (pentane/ $\mathrm{Et}_{2} \mathrm{O}$ : 2/1) 0.13; mp 158-160 ${ }^{\circ} \mathrm{C}$; IR $v 2912,2844,1719,1587,1464$; ${ }^{1} \mathrm{H}$ NMR $\delta 8.34,7.80$ (2d, $J=8.9$, 2H, ArH), 6.76 (s, $1 \mathrm{H}, \mathrm{CH}=\mathrm{C}$ ), 4.56 (dd, $J=6.8,2.2,1 \mathrm{H}, \mathrm{CHBr}), 3.82-3.73,3.41-3.34\left(2 \mathrm{~m}, 2 \mathrm{H}, \mathrm{CH}_{2}\right) ;{ }^{13} \mathrm{C}$ NMR $\delta$ 201.0 (CO), $167.4(\mathrm{CH}=\mathrm{C}), 138.7,127.6$ (ArC), 127.8, 124.3 ( $\mathrm{ArCH}, \mathrm{CH}=\mathrm{C}), 40.2(\mathrm{CHBr}), 29.6$ 
$\left(\mathrm{CH}_{2}\right) ; \mathrm{MS} m / z 281\left(\mathrm{M}^{+}+1,1.70\right), 204\left(\mathrm{M}^{+}+2-\mathrm{Br}, 10\right), 203\left(\mathrm{M}^{+}+1-\mathrm{Br}, 83\right), 202\left(\mathrm{M}^{+}-\mathrm{Br}, 10\right), 187$ (12), 186 (60), 157 (19), 156 (77), 129 (32), 128 (100), 127 (37), 115 (26), 102 (17), 101 (15), 89 (14), 77 (13), 75 (19), 63 (16), 51 (19); HRMS Calcd for $\mathrm{C}_{11} \mathrm{H}_{8} \mathrm{BrNO}_{3}\left(\mathrm{M}^{+}\right)$280.9681, 202.0599 ( $\mathrm{M}^{+}-\mathrm{Br}$ ) found: 202.0499 .

Isopropyl 4-azido-3-oxo-1-cyclopentenecarboxylate (14). Colorless oil; $\mathrm{R}_{f}$ (pentane/ $\mathrm{Et}_{2} \mathrm{O}: 4 / 1$ ) 0.31; IR $v$ 2986, 2931, 2253, 2116, 1731, 1231, 1107, 910, 732; ${ }^{1} \mathrm{H}$ NMR $\delta 6.75$ (s, $\left.1 \mathrm{H}, \mathrm{CH}=\mathrm{C}\right)$, 5.17 (sept., $J=6.2,1 \mathrm{H}, \mathrm{OCH}$ ), 4.14 (dd, $J=7.3,3.1,1 \mathrm{H}, \mathrm{CHN}_{3}$ ), 3.23-3.14, 2.63-2.55 (2m, 2H, $\left.\mathrm{CH}_{2}\right), 1.33\left(\mathrm{~d}, J=6.2,6 \mathrm{H}, 2 \times \mathrm{CH}_{3}\right) ;{ }^{13} \mathrm{C}$ NMR $\delta 204.2(\mathrm{CO}), 168.8,168.4\left(\mathrm{CO}_{2} \mathrm{Pr}^{\mathrm{i}}, \mathrm{CH}=C\right), 135.1$ $(\mathrm{CH}=\mathrm{C}), 70.0(\mathrm{OCH}), 60.6\left(\mathrm{CHN}_{3}\right), 33.8\left(\mathrm{CH}_{2}\right), 21.6\left(2 \times \mathrm{CH}_{3}\right)$; $\mathrm{MS} \mathrm{m} / z 209\left(\mathrm{M}^{+}, 0.86\right), 167(32)$, 150 (11), 149 (100), 71 (15), 70 (14), 57 (23); HRMS Calcd for $\mathrm{C}_{9} \mathrm{H}_{11} \mathrm{~N}_{3} \mathrm{O}_{3}\left(\mathrm{M}^{+}\right)$209.0800, $\left(\mathrm{M}^{+}-\mathrm{N}_{2}\right)$ 181.0739, found: 181.0746

\section{Acknowledgements}

This work has been supported by the Dirección General de Investigación of the Ministerio de Educación y Ciencia (CTQ2004-00808/BQU), by the Generalitat Valenciana (CTIOIB/2002/320, GRUPOS03/134, GRUPOS05/11 and GV05/157) and the University of Alicante. M. F. thanks the University of Alicante for a predoctoral fellowship.

\section{References and Notes}

1. Mahrwald, R., Ed. Modern Aldol Reactions; Wiley-VCH: Weinheim, 2004.

2. Becker, K. B. Tetrahedron 1980, 36, 1717.

3. Kirmse, W. Angew. Chem. Int. Ed. 1997, 36, 1164.

4. Nazarov, I. N.; Zaretskaya, I. I. Izv. Akad. Nauk. SSSR, Ser. Khim. 1941, 211. (b) Frontier, A. J.; Collison, C. Tetrahedron 2005, 61, 7577.

5. Gibson, S. E.; Lewis, S. E.; Mainolfi, N. J. Organomet. Chem. 2004, 689, 3873.

6. Khand, I. U.; Knox, G. R.; Pauson, P. L.; Watts, W. E.; Foreman, M. I. J. Chem. Soc., Perkin Trans. 1 1973, 977. (b) Brummond, K. M.; Kent, J. L. Tetrahedron 2000, 56, 3263.

7. Rautenstrauch, V. J. Org. Chem. 1984, 49, 950.

8. Alonso, D. A.; Nájera, C.; Varea, M. Tetrahedron Lett. 2001, 42, 8845. (b) Alonso, D. A.; Nájera, C.; Varea, M. Helv. Chim. Acta 2003, 85, 4287.

9. Alonso, D. A.; Nájera, C.; Varea, M. Synthesis 2003, 277.

10. Blakemore, P. R. J. Chem. Soc., Perkin Trans. 1 2002, 2563.

11. (a) Alonso, D. A.; Nájera, C.; Varea, M. Tetrahedron Lett. 2004, 45, 573. (b) Alonso, D. A.; Fuensanta, M.; Nájera, C.; Varea, M. Phosphorus, Sulfur Silicon Relat. Elem. 2005, 180, 1119. (c) Alonso, D. A.; Fuensanta, M.; Nájera, C.; Varea, M. J. Org. Chem. 2005, 70, 6404. (d) Alonso, D. A.; Fuensanta, M.; Nájera, C. Eur. J. Org. Chem. 2006, 4747.

12. For a previous report on the synthesis of 3,5-disubstituted cyclopentenones employing sulfone linkers, see: Cheng, W. C.; Kurth, M. J. J. Org. Chem. 2002, 67, 4387.

13. Alonso, D. A.; Nájera, C. In Electronic Encyclopedia of Reagents for Organic Synthesis, Paquette, L.A., Ed.; Wiley, 2005. 
14. This is a simple, "green" and general method for the oxidation of sulfides to sulfones: Alonso, D. A.; Nájera, C.; Varea, M. Tetrahedron Lett. 2002, 43, 3459.

15. (a) Fuchs, P. L.; Nantz, M. H. J. Org. Chem. 1987, 52, 5298. (b) Blake, A. J.; Cooke, P. A.; Kendall, J. E.; Simpkins, N. S.; Westaway, S. M. J. Chem. Soc., Perkin Trans. 1 2000, 153.

16. Das, B.; Krishnaiah, M.; Venkateswarlu, K. Tetrahedron Lett. 2006, 47, 4457.

17. Ley, S. V.; Norman, J.; Griffith, W. P.; Marsden, S. P. Synthesis 1994, 639.

18. For a recent review on homogeneous transition metal-catalyzed aerobic alcohol oxidations, see: Schultz, M. J.; Sigman, M. S. Tetrahedron 2006, 62, 8227.

19. (a) Frigerio, M.; Santagostino, M.; Sputore, S. J. Org. Chem. 1999, 64, 4537. (b) Wirth, T. Angew. Chem. Int. Ed. 2001, 40, 2812. (c) Nicolau, K. C.; Montagnon, T.; Baran, P. S.; Zhong, Y. L. J. Am. Chem. Soc. 2002, 124, 2245.

20. Dess, D. B.; Martin, J. C. J. Am. Chem. Soc. 1991, 113, 7277. 